\title{
Parameters Sensitivity Characteristics of Highly Integrated Valve-Controlled Cylinder Force Control System
}

\author{
Kai-Xian Ba ${ }^{1}$, Bin Yu ${ }^{1,2^{*}}$, Xiang-Dong Kong ${ }^{1}$, Chun-He Li ${ }^{1}$, Qi-Xin Zhu' ${ }^{1}$, Hua-Long Zhao ${ }^{1}$ and Ling-Jian Kong ${ }^{3}$
}

\begin{abstract}
Nowadays, a highly integrated valve-controlled cylinder (HIVC) is applied to drive the joints of legged robots. Although the adoption of HIVC has resulted in high-performance robot control, the hydraulic force system still has problems, such as strong nonlinearity, and time-varying parameters. This makes HIVC force control very difficult and complex. How to improve the control performance of the HIVC force control system and find the influence rule of the system parameters on the control performance is very significant. Firstly, the mathematical model of HIVC force control system is established. Then the mathematical expression for parameter sensitivity matrix is obtained by applying matrix sensitivity analysis (PSM). Then, aimed at the sinusoidal response under (three factors and three levels) working conditions, the simulation and the experiment are conducted. While the error between the simulation and experiment can't be avoided. Therefore, combined with the range analysis, the error in the two performance indexes of sinusoidal response under the whole working condition is analyzed. Besides, the sensitivity variation pattern for each system parameter under the whole working condition is figured out. Then the two sensitivity indexes for the three system parameters, which are supply pressure, proportional gain and initial displacement of piston, are proved experimentally. The proposed method significantly reveals the sensitivity characteristics of HIVC force control system, which can make the contribution to improve the control performance.
\end{abstract}

Keywords: Highly integrated valve-controlled cylinder system, Force control system, Matrix sensitivity analysis, Orthogonal test design, Sensitivity index

\section{Introduction}

The valve-controlled cylinder system is the most commonly used component in the hydraulic system and is widely used in many fields such as aerospace, metallurgy, engineering machinery, agricultural machinery and advanced manufacturing. The highly integrated valvecontrolled cylinder (HIVC) which has the high power density is used with unique advantages in the aerospace load simulator and the high performance legged robot which are different from the traditional civil equipment and require higher entire performance to ensure better adaptability under the complex environment. Therefore,

\footnotetext{
*Correspondence: yb@ysu.edu.cn

${ }^{1}$ School of Mechanical Engineering, Yanshan University,

Qinhuangdao 066004, China

Full list of author information is available at the end of the article
}

the lighter weight and the faster response are required for the HIVC, which makes the structure optimization and the compensation control method of the HIVC have practical significance [1-5].

The control methods of the HIVC include the position control and the force control, and the position control compared with the force control is more mature, yet the force control is widely applied to the heavy industry, the aerospace load simulator and the advanced manufacturing. But the quantitative performance analysis and the system optimization method of the force control are less, and how to improve the force control performance effectively should be analyzed further. The force control is relied on the compressibility of the oil and is achieved through controlling the oil volume of the closed oil cavity, and ensuring the stability of the control component with small valve opening increases the force control
Springer Open

(c) The Author(s) 2018. This article is distributed under the terms of the Creative Commons Attribution 4.0 International License (http://creativecommons.org/licenses/by/4.0/), which permits unrestricted use, distribution, and reproduction in any medium, provided you give appropriate credit to the original author(s) and the source, provide a link to the Creative Commons license, and indicate if changes were made. 
difficulty and makes the effect of each system parameter on the force control system performance more sensitive. There are many parameters in the hydraulic system including structure parameters, working parameters and control parameters whose uncertainty caused by the manufacturing error, the testing error, the material aging, the non-ideal working condition and the online working parameters fluctuating makes the real working performance no complete accordance to the ideal working performance, which is involved with what effect degree of the parameter change has on the force control performance. Therefore, it is needed to research which parameter has greater effects on the force control performance and should be optimized and compensated and which parameter has smaller effects and can be ignored in the compensation control and structure optimization. The sensitivity analysis theory just can be applied to research the parameters change effects on the hydraulic system dynamic characteristics, and the sensitivity analysis results of the HIVC can be used to improve the system working performance, which would provide a novel idea for analyzing the system performance quantitatively and optimizing the compensation control method effectively, so the sensitivity analysis of HIVC force control system has significant theoretical research value and application prospects.

There are many sensitivity analysis methods including the matrix sensitivity analysis (MSA), the trajectory sensitivity analysis, the global sensitivity analysis, the variance-based sensitivity analysis and so on. All these sensitivity analysis methods have different mathematical operation characteristics, so the operation accuracy, the operation style and the operation process are different, which makes each sensitivity analysis method have its own advantages and applied range. The MSA is based on the matrix operation that is applicable to the higher order hydraulic system even involved with many nonlinear problems. Therefore, the MSA is adopted to analyze the parameter sensitivity of the HIVC force control system. In recent years, the different sensitivity analysis methods are applied to different fields. Ya et al. [6] adopted to trajectory sensitivity analysis research the correlation coefficients of random variables of mechanical structures, which provided guidance for the system reliability researches. Abolfaz et al. [7] adopted trajectory sensitivity analysis to research the stability of underground rock structures, finding deep-influencing geo mechanical parameters. Liu et al. [8] adopted trajectory sensitivity analysis to research the structure parameters of the engine pipelines and demonstrated that some supports' positions played more important roles for complex piping, and the engine pipelines structure parameters were optimized. Chatterjee et al. [9] adopted trajectory sensitivity analysis to found series and shunt compensators in order to improve the transient stability of power system. Song et al. [10] adopted global sensitivity analysis to research the key parameters that affect model performance, and analyzed relationship between parameter identification, uncertainty analysis, and optimization in hydrological modeling. Zheng et al. [11] adopted global sensitivity analysis to perform on a marine ecosystem dynamic model, and proposed the model's parameter optimization strategy and applied it to the Sanggou Bay. Saltelli et al. [12] adopted variance-based sensitivity analysis to research the various available techniques for sensitivity analysis of model output. Hall et al. [13] adopted variance-based sensitivity analysis to give an incomplete picture of model response over the range of variability in the hydraulic model inputs, and shown that the variancebased sensitivity analysis is more general in its applicability and in its capacity to reflect nonlinear processes and the effects of interactions among variables. Bashar et al. [14] adopted MSA to research the performance difference between CPU and GPU in computer system. Billy et al. [15] adopted MSA to solve the steady-state operating point of regulator circuits and also examine the stability of operation in power system, and other methods are compared in the performance of the steadystate determination and stability analysis. Zhang et al. [16] adopted MSA to research the electrical impedance tomography by using the sensitivity matrix update, and the reconstructed images after sensitivity matrix update were compared in power system. Naseralavi et al. [17] adopted MSA to research the structural damage detection of cyclic structures.

Yet sensitivity analysis has a few applications in hydraulic system. Vilenus [18] applied trajectory sensitivity analysis to the valve-controlled cylinder system of the logging machine and the mathematical model of this system was simplified to fifth order model where the servo valve flow-pressure nonlinearity and dead zone nonlinearity were taken into consideration. Moreover, the sensitivity analysis of ten main parameters containing viscous damping coefficient, conversion mass, internal leakage coefficient of servo cylinder, effective piston area of servo cylinder, effective bulk modulus, servo valve gain, nature frequency of servo valve, damping ratio of servo valve, conversion coefficient and system supply oil pressure is obtained with parameters change $1 \%$ under single working condition. The sensitivity analysis results indicated that the sensitivity of piston rod area of the servo cylinder, servo valve gain, conversion flow coefficient and system supply oil pressure is large and that of viscous damping coefficient, internal leakage coefficient of the servo valve, damping ratio of servo vale and nature frequency of the servo valve is relatively small under the 
same working condition. Furthermore, Farasat et al. [19] founded the fourth order linear mathematical model of the valve-controlled cylinder position control system where the servo valve flow-pressure nonlinearity is linearized partially. The sensitivity of other parameters, such as servo valve torque motor constant, servo valve area constant, orifice opening of metering valve, system return oil pressure, supply oil volume, return oil volume and input signal voltage, is given with parameters change $1 \%$ under single working condition by four sensitivity evaluation methods including VILENUS method, revised VILENUS method, Entirety index method and Individual characteristics method. The analysis results indicated that the sensitivity of effective piston area of servo cylinder, flow coefficient, conversion mass and input signal voltage is relatively small. Kong et al. [20-22] first established the fifth linear order mathematical model for the hydraulic drive unit of quadruped robot and derived the first order trajectory sensitivity formulas analysis. Aimed at the imprecise conclusion of sensitivity analysis obtained from the linear model, some nonlinear models are also established. Besides, the sensitivity mathematical expressions with nonlinear and time-varied parameters are derived under the trotting gait working conditions. Kong et al. [23] derived the mathematical model of the second order trajectory sensitivity analysis based on the mathematical model of the first order trajectory sensitivity formulas analysis. Therefore, the situation where the first order trajectory sensitivity formulas analysis accuracy of some parameters is not high is improved. Then the experimental verification is conducted.

In Refs. [18-22], the hydraulic system sensitivity analysis was adopted to analyze the position control system and the mathematical model was simple even some of them.The trajectory sensitivity analysis method had the disadvantage of large computation, and the sensitivity results are obtained with only position step signal under one single working condition, which relatively decreased the reference value of the sensitivity results. However, the force control system is one of the main control methods of the HIVC and many problems need adopt the sensitivity analysis to solve. What is the sensitivity change rule of the force control parameters? How can the system parameters change influence the output response when the input signal is sinusoidal? What can the effects of each system parameter change have on the two performance indexes of sinusoidal response that are the amplitude attenuation and the phase lag which reflect on the control accuracy and response speed respectively? Which parameters change can improve or restrain the force control performance? How can the system parameters

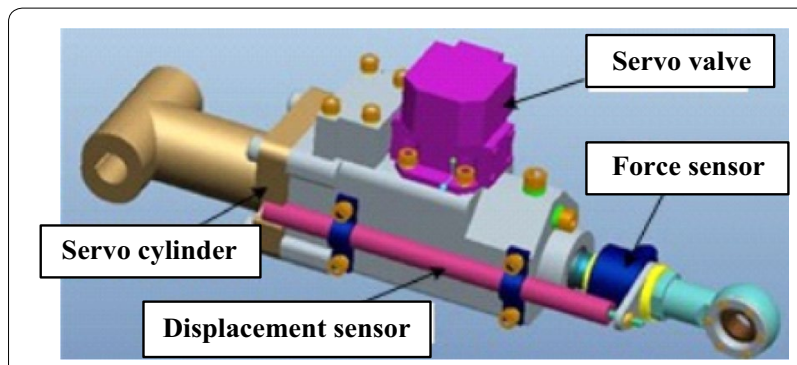

Figure 1 Three-dimensional assembly drawing of HIVC

sensitivity change under different working conditions? Especially, are the experimental results and the theoretical analysis results coincident? With the above issues this paper is organized as follows: Firstly, the force control system mathematical model and the experimental system are described. Secondly, the MSA common mathematical model and the appropriate MSA mathematical model for the HIVC are derived. Thirdly, working conditions are listed and nine working conditions based on the orthogonal test design are selected to conduct the simulation analysis and the experiment research, and the dynamic change of the system parameters sensitivity is researched under these nine selected working conditions. Fourthly, the two sensitivity indexes based on the two performance indexes of sinusoidal response are derived, and the range analysis table based on the two sensitivity indexes is built to analyze the sensitivity value and change rules quantitatively under the whole twenty-seven working conditions. Moreover, the effects of each parameter on the force control performance and the methods to improve the force control performance are concluded. Finally, the sensitivity analysis results are verified experimentally.

\section{Introduction of HIVC}

\subsection{System Compositions}

In this paper, the three-dimensional assembly drawing of HIVC is shown in Figure 1 [24].

The performance test platform schematic of HIVC is shown in Figure 2.

The performance test platform photo of HIVC is shown in Figure 3.

In Figures 2 and 3, the right channel which consists of small servo valve and servo cylinder is the tested HIVC controlled by the force-loop method, while the left channel which consists of the same type servo valve and servo cylinder is the position simulation part controlled by the position-loop method. The two channels are connected by the cylinder piston rod, and displacement sensor and force sensor are installed in their joint. 


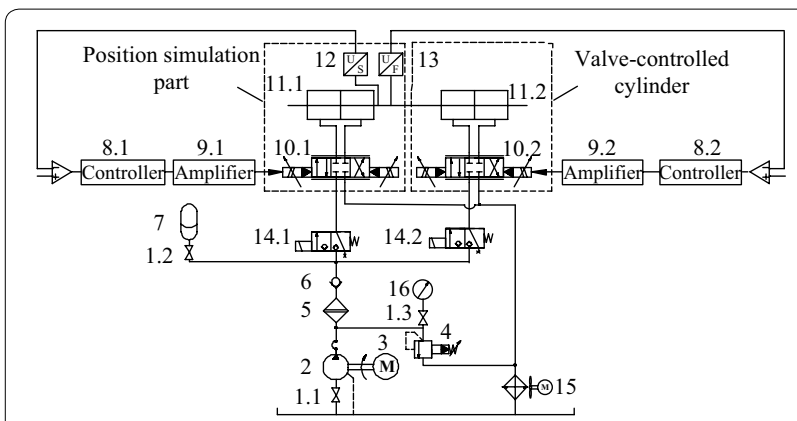

Figure 2 Performance test platform schematic of HIVC. 1. Globe valve; 2 . Axial piston pump; 3. Electromotor; 4. Relief valve 5. Highpressure filter; 6 . Check valve; 7. Accumulator; 8 . dSPACE controller; 9. Servo valve amplifier; 10 . Servo valve; 11 . Servo cylinder; 12 . Displacement sensor; 13. Force sensor; 14. Electromagnetic valve; 15. Cooler; 16. Pressure meter

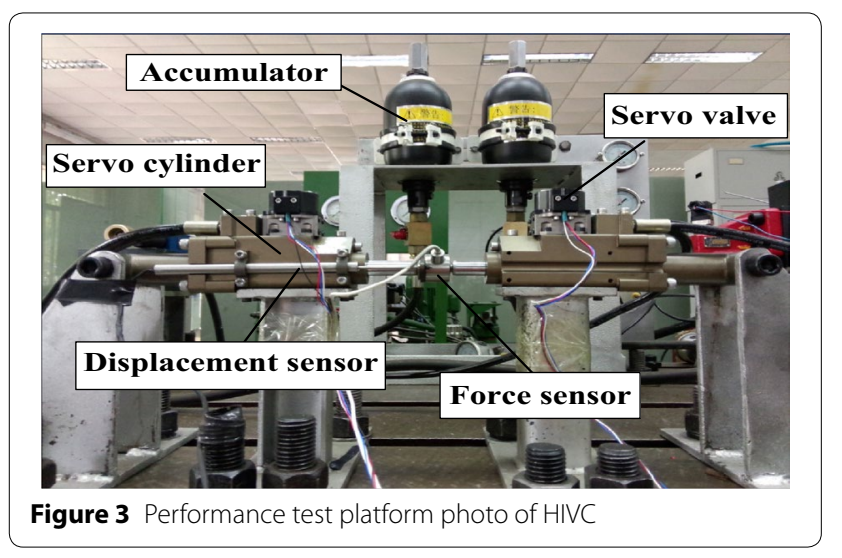

\subsection{Mathematical Model of HIVC Force Control System}

The force control system transfer block diagram of HIVC is shown in Figure 4.

In Figure $4, K_{\mathrm{d}}=C_{\mathrm{d}} W \sqrt{2 / \rho}, \omega$ is natural frequency of servo valve, $\zeta$ is damping ratio of servo valve, $C_{\mathrm{d}}$ is orifice flow coefficient of spool valve, $W$ is area gradient of spool valve, $\rho$ is density of hydraulic oil, $K_{\mathrm{d}}$ is conversion coefficient, $p_{\mathrm{s}}$ is system supply oil pressure, $p_{0}$ is system return oil pressure, $p_{1}$ is inlet cavity pressure of servo cylinder, $p_{2}$ is outlet cavity pressure of servo cylinder, $C_{\mathrm{ip}}$ is internal leakage coefficient of servo cylinder, $C_{\mathrm{ep}}$ is external leakage coefficient of servo cylinder, $L$ is total piston stroke of servo cylinder, $L_{0}$ is initial piston position of servo cylinder, $A_{\mathrm{p}}$ is effective piston area of servo cylinder, $\beta_{\mathrm{e}}$ is effective bulk modulus, $m_{\mathrm{t}}$ is conversion mass(including the piston, the displacement sensor, the force sensor, the connecting pipe and the oil in servo cylinder), $x_{\mathrm{p}}$ is servo cylinder piston displacement, $x_{\mathrm{v}}$ is servo valve spool displacement, $x_{\mathrm{r}}$ is input displacement, $K_{\mathrm{axv}}$ is servo valve gain, $K_{\mathrm{f}}$ is force sensor gain, $K_{\mathrm{p}}$ is proportional gain, $K$ is load stiffness, $B_{\mathrm{p}}$ is viscous damping coefficient, $F$ is output force, $F_{\mathrm{L}}$ is external load force, $V_{\mathrm{g} 1}$ is volume of input oil pipe, $V_{\mathrm{g} 2}$ is volume of output oil pipe, $F_{\mathrm{f}}$ is friction, $U_{\mathrm{r}}$ is input voltage, $U_{\mathrm{p}}$ is displacement sensor feedback voltage, $U_{\mathrm{g}}$ is controller output voltage, $q_{1}$ is inlet oil flow, $q_{2}$ is outlet oil flow. It can be seen in Figure 4 that the highest order of system transfer block diagram is sixth, and denote each vector in Table 1.

In Table 1, the state vector containing six state variables, input vector containing one input and parameter vector containing fourteen key parameters are selected, Denote the expanded form of the state space Eq. (1) including vectors in Table 1 as follows:

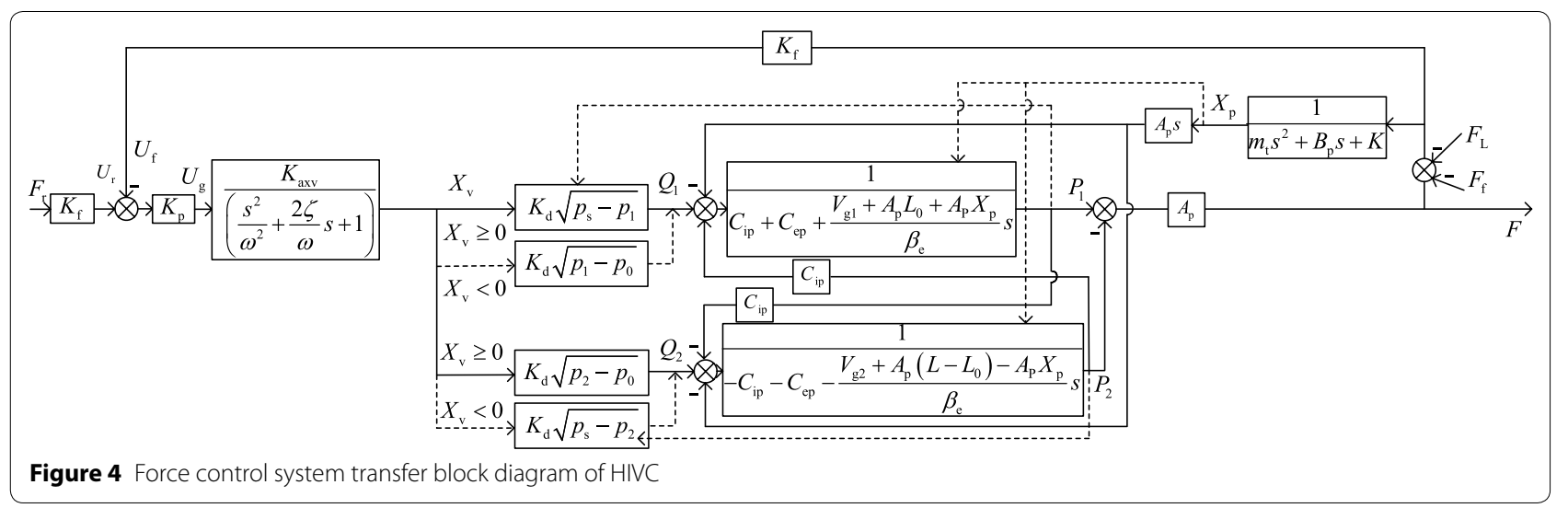


Table 1 Each vector of the state space equations

\begin{tabular}{lllll}
\hline State vector & Input vector & Output vector & \multicolumn{2}{c}{ Parameter vector } \\
\hline$x_{1}=x_{p}$ & $u_{1}=F_{r}$ & $Y=F$ & $\alpha_{1}=\omega$ & $\alpha_{8}=A_{p}$ \\
$x_{2}=\dot{x}_{p}$ & & & $\alpha_{2}=\zeta$ & $\alpha_{9}=\beta_{e}$ \\
$x_{3}=x_{v}$ & & $\alpha_{3}=p_{s}$ & $\alpha_{10}=m_{t}$ \\
$x_{4}=\dot{x}_{v}$ & & $\alpha_{4}=p_{0}$ & $\alpha_{11}=K_{f}$ \\
$x_{5}=p_{1}$ & & $\alpha_{5}=C_{i p}$ & $\alpha_{12}=K_{p}$ \\
$x_{6}=p_{2}$ & & $\alpha_{6}=L$ & $\alpha_{13}=K$ \\
& & $\alpha_{7}=L_{0}$ & $\alpha_{14}=B_{p}$ \\
\hline
\end{tabular}

$$
\left\{\begin{aligned}
\dot{x}_{1}= & x_{2}, \\
\dot{x}_{2}= & -\frac{K}{m_{\mathrm{t}}} x_{1}-\frac{B_{\mathrm{p}}}{m_{\mathrm{t}}} x_{2}+\frac{A_{\mathrm{p}}}{m_{\mathrm{t}}} x_{5}-\frac{A_{\mathrm{p}}}{m_{\mathrm{t}}} x_{6}-\frac{F_{\mathrm{f}}+F}{m_{\mathrm{t}}}, \\
\dot{x}_{3}= & x_{4}, \\
\dot{x}_{4}= & -K_{\mathrm{f}} K_{\mathrm{axv}} K_{\mathrm{p}} \omega^{2}\left[A_{\mathrm{p}}\left(x_{5}-x_{6}\right)-F_{\mathrm{f}}-F\right]- \\
& \omega^{2} x_{3}-2 \zeta \omega x_{4}+K_{\mathrm{axv}} K_{\mathrm{p}} K_{\mathrm{f}} \omega^{2} u_{1}, \\
\dot{x}_{5}= & \beta_{\mathrm{e}}\left(V_{\mathrm{g} 1}+A_{\mathrm{p}} L_{0}+A_{\mathrm{p}} x_{2}\right)^{-1}\left[-A_{\mathrm{p}} x_{2}+\right. \\
& K_{\mathrm{d}} x_{3} \sqrt{\left\{\frac{\left[1+\operatorname{sgn}\left(x_{3}\right)\right] p_{\mathrm{s}}}{2}+\frac{\left[-1+\operatorname{sgn}\left(x_{3}\right)\right] p_{0}}{2}\right\}-\operatorname{sgn}\left(x_{3}\right) x_{5}-} \\
& \left.\left(C_{\mathrm{ip}}+C_{\mathrm{ep}}\right) x_{5}+C_{\mathrm{ip}} x_{6}\right], \\
\dot{x}_{6}= & \beta_{\mathrm{e}}\left(V_{\mathrm{g} 2}+A_{\mathrm{p}}\left(L-L_{0}\right)-A_{\mathrm{p}} x_{2}\right)^{-1}\left[A_{\mathrm{p}} x_{2}-\right. \\
& K_{\mathrm{d}} x_{3} \sqrt{\left\{\frac{\left[1-\operatorname{sgn}\left(x_{3}\right)\right] p_{\mathrm{s}}}{2}+\frac{\left[-1-\operatorname{sgn}\left(x_{3}\right)\right] p_{0}}{2}\right\}+\operatorname{sgn}\left(x_{3}\right) x_{6}+} \\
& \left.C_{\mathrm{ip}} x_{5}-\left(C_{\mathrm{ip}}+C_{\mathrm{ep}}\right) x_{6}\right], \\
Y= & A_{\mathrm{p}}\left(x_{5}-x_{6}\right) .
\end{aligned}\right.
$$

For the friction relative to the output force is small, so it is ignored in this paper. And denote the system equations including vectors in Table 1 as follows:

$$
\begin{aligned}
& \boldsymbol{g}(\boldsymbol{x}, \boldsymbol{u}, \boldsymbol{\alpha}, t)=\mathbf{0} \\
& =\left\{\begin{array}{l}
\dot{x}_{1}-x_{2}, \\
\dot{x}_{2}+\frac{K}{m_{\mathrm{t}}} x_{1}+\frac{B_{\mathrm{p}}}{m_{\mathrm{t}}} x_{2}-\frac{A_{\mathrm{p}}}{m_{\mathrm{t}}} x_{5}+\frac{A_{\mathrm{p}}}{m_{\mathrm{t}}} x_{6}+\frac{F_{\mathrm{f}}+F}{m_{\mathrm{t}}} \\
\dot{x}_{3}-x_{4} \\
\dot{x}_{4}+K_{\mathrm{f}} K_{\mathrm{axv}} K_{\mathrm{p}} \omega^{2}\left[A_{\mathrm{p}}\left(x_{5}-x_{6}\right)-F_{\mathrm{f}}-F\right]- \\
\omega^{2} x_{3}-2 \zeta \omega x_{4}+K_{\mathrm{axv}} K_{\mathrm{p}} K_{\mathrm{f}} \omega^{2} u_{1}, \\
\dot{x}_{5}-\beta_{\mathrm{e}}\left(V_{\mathrm{g} 1}+A_{\mathrm{p}} L_{0}+A_{\mathrm{p}} x_{2}\right)^{-1}\left[-A_{\mathrm{p}} x_{2}+\right. \\
K_{\mathrm{d}} x_{3} \sqrt{\left\{\frac{\left[1+\operatorname{sgn}\left(x_{3}\right)\right] p_{\mathrm{s}}}{2}+\frac{\left[-1+\operatorname{sgn}\left(x_{3}\right)\right] p_{0}}{2}\right\}-\operatorname{sgn}\left(x_{3}\right) x_{5}-} \\
\left.\left(C_{\mathrm{ip}}+C_{\mathrm{ep}}\right) x_{5}+C_{\mathrm{ip}} x_{6}\right], \\
\dot{x}_{6}-\beta_{\mathrm{e}}\left(V_{\mathrm{g} 2}+A_{\mathrm{p}}\left(L-L_{0}\right)-A_{\mathrm{p}} x_{2}\right)^{-1}\left[A_{\mathrm{p}} x_{2}-\right. \\
K_{\mathrm{d}} x_{3} \sqrt{\left\{\frac{\left[1-\operatorname{sgn}\left(x_{3}\right)\right] p_{\mathrm{s}}}{2}+\frac{\left[-1-\operatorname{sgn}\left(x_{3}\right)\right] p_{0}}{2}\right\}+\operatorname{sgn}\left(x_{3}\right) x_{6}+} \\
\left.C_{\mathrm{ip}} x_{5}-\left(C_{\mathrm{ip}}+C_{\mathrm{ep}}\right) x_{6}\right] .
\end{array}\right.
\end{aligned}
$$

Table 2 Simulation model parameters and initial value

\begin{tabular}{ll}
\hline Simulation model parameters & Initial value \\
\hline Servo valve gain $K_{\mathrm{axv}}(\mathrm{mm} / \mathrm{v})$ & 0.45 \\
Natural frequency of servo valve $\omega(\mathrm{rad} / \mathrm{s})$ & 628 \\
Damping ratio of servo valve $\zeta$ & 0.77 \\
Effective piston area of servo cylinder $A_{\mathrm{p}}\left(\mathrm{mm}^{2}\right)$ & 336.8 \\
Volume of input oil pipe $V_{\mathrm{g} 1}\left(\mathrm{~mm}^{3}\right)$ & 620 \\
Volume of output oil pipe $V_{\mathrm{g} 2}\left(\mathrm{~mm}^{3}\right)$ & 860 \\
Total piston stroke of servo cylinder $L(\mathrm{~mm})$ & 50 \\
Initial piston position of servo cylinder $L_{0}(\mathrm{~mm})$ & 20 \\
System return oil pressure $p_{0}(\mathrm{MPa})$ & 0.5 \\
Proportional gain $K_{\mathrm{p}}$ & 8 \\
Force sensor gain $K_{\mathrm{f}}(\mathrm{V} / \mathrm{kN})$ & 0.0769 \\
External leakage coefficient $C_{\mathrm{ep}}\left(\mathrm{mm}^{3} \cdot(\mathrm{s} \cdot \mathrm{MPa})^{-1}\right)$ & 0 \\
Internal leakage coefficient $C_{\mathrm{ip}}\left(\mathrm{mm}{ }^{3} \cdot(\mathrm{s} \cdot \mathrm{MPa})^{-1}\right)$ & 238 \\
Conversion mass $m_{\mathrm{t}}(\mathrm{kg})$ & 1.1315 \\
Effective bulk modulus $\mathrm{B}^{6} \mathrm{MPa}$ & 800 \\
Load stiffness $K(\mathrm{kN} / \mathrm{m})$ & 450 \\
Viscous damping coefficient $B_{\mathrm{p}}\left(\mathrm{kN} /\left(\mathrm{m} \cdot \mathrm{s}^{-1}\right)\right)$ & 2 \\
Conversion coefficient $K_{\mathrm{d}}\left(\mathrm{mm}{ }^{2} / \mathrm{s}\right)$ & 124.8 \\
\hline
\end{tabular}

The servo valve natural parameters are obtained from the servo valve product book which shows the fitted time domain and frequency domain characteristic curves. The structure parameters including effective piston area of servo cylinder, total piston stroke of servo cylinder and the volume of oil pipe are the factory data of the cylinder. The working parameters including system supply oil pressure, system return oil pressure and sensor gain are taken from the experiment test, while other parameters are selected through engineering experience. Therefore, the force control system parameters and initial value of HIVC are shown in Table 2.

\section{MSA Mathematical Model}

3.1 MSA Mathematical Common Model

Denote the common system equations as follows:

$$
\boldsymbol{g}(\boldsymbol{x}, \boldsymbol{u}, \alpha, t)=\mathbf{0},
$$

where $\boldsymbol{x}$ is $m$-dimensional state vector, $\boldsymbol{u}$ is $r$-dimensional vector unrelated to $\boldsymbol{\alpha}, \boldsymbol{\alpha}$ is $p$-dimensional vector and $t$ is time.

Initial value of the state vector $\boldsymbol{x}_{0}$ can be obtained by giving initial value of the input vector $\boldsymbol{u}_{0}$ and the initial value of parameter vector $\boldsymbol{\alpha}_{0}$, and initial state of Eq. (3) are

$$
\boldsymbol{g}\left(\boldsymbol{x}_{0}, \boldsymbol{u}_{0}, \alpha_{0}, t\right)=\mathbf{0}
$$


Denote Eq. (4) where input vector change $\Delta \boldsymbol{u}$ and parameter vector change $\Delta \boldsymbol{\alpha}$ causes the state vector change $\Delta \boldsymbol{x}$ as follows:

$$
\boldsymbol{g}\left(\boldsymbol{x}_{0}+\Delta \boldsymbol{x}, \boldsymbol{u}_{0}+\Delta \boldsymbol{u}, \boldsymbol{\alpha}_{0}+\Delta \boldsymbol{\alpha}, t\right)=\mathbf{0} .
$$

Denote the Taylor expansion of Eq. (5) whose high order Taylor expansions are ignored as follows:

$$
\begin{aligned}
& \boldsymbol{g}\left(\boldsymbol{x}_{0}+\Delta \boldsymbol{x}, \boldsymbol{u}_{0}+\Delta \boldsymbol{u}, \boldsymbol{\alpha}_{0}+\Delta \boldsymbol{\alpha}, t\right)=\boldsymbol{g}\left(\boldsymbol{x}_{0}, \boldsymbol{u}_{0}, \boldsymbol{\alpha}_{0}, t\right) \\
& \quad+\boldsymbol{g}_{x} \cdot \Delta \boldsymbol{x}+\boldsymbol{g}_{u} \cdot \Delta \boldsymbol{u}+\boldsymbol{g}_{\alpha} \cdot \Delta \boldsymbol{\alpha}=\mathbf{0} .
\end{aligned}
$$

Eq. (4) are taken into Eq. (6), the following is

$$
\boldsymbol{g}_{x} \cdot \Delta \boldsymbol{x}+\boldsymbol{g}_{u} \cdot \Delta \boldsymbol{u}+\boldsymbol{g}_{\alpha} \cdot \Delta \boldsymbol{\alpha}=\mathbf{0} .
$$

Eq. (7) can be turned into

$$
\Delta \boldsymbol{x}=-\boldsymbol{g}_{x}^{-1} \cdot \boldsymbol{g}_{u} \cdot \Delta \boldsymbol{u}-\boldsymbol{g}_{x}^{-1} \cdot \boldsymbol{g}_{\alpha} \cdot \Delta \boldsymbol{\alpha} .
$$

Define

$$
\boldsymbol{S}_{\alpha}=\boldsymbol{g}_{x}^{-1} \cdot \boldsymbol{g}_{\alpha},
$$

and

$$
\boldsymbol{S}_{\mathrm{u}}=\boldsymbol{g}_{x}^{-1} \cdot \boldsymbol{g}_{u} .
$$

Eqs. (9) and (10) are taken into Eq. (8), the following is

$$
\Delta x=-S_{\mathrm{u}} \cdot \Delta \boldsymbol{u}-S_{\alpha} \cdot \Delta \alpha,
$$

where $S_{\alpha}$ is $m \times p$ order parameter sensitivity matrix (PSM) which contains time-varied elements, and $S_{\mathrm{u}}$ is $m \times r$ order input sensitivity matrix (ISM) which also contains time-varied elements.

Denote the system output vector change as follows:

$\Delta \boldsymbol{Y}=\boldsymbol{C} \cdot \Delta \boldsymbol{x}+\boldsymbol{D}=\boldsymbol{C} \cdot\left(-\boldsymbol{S}_{\mathrm{u}} \cdot \Delta \boldsymbol{u}-\boldsymbol{S}_{\alpha} \cdot \Delta \boldsymbol{\alpha}\right)+D$.

Based on Eq. (12) which is derived by MSA, the approximate result of the output vector change $\Delta Y$ caused by the parameter vector change $\Delta \alpha$ and input vector change $\Delta \boldsymbol{u}$ can be obtained after the PSM $\boldsymbol{S}_{\alpha}$ and ISM $\boldsymbol{S}_{\mathrm{u}}$ are taken into Eq. (12).

\subsection{MSA Mathematical Model of HIVC}

In HIVC, the matrix in Eq. (7) is $\partial \boldsymbol{g} / \partial \boldsymbol{x}$, which can be expressed $6 \times 6$ order Jacobian matrix as follows:

$$
\boldsymbol{g}_{x}=\left(\begin{array}{cccc}
a_{1,1} & a_{1,2} & \cdots & a_{1,6} \\
a_{2,1} & a_{2,2} & & \\
\vdots & & \ddots & \vdots \\
a_{6,1} & \cdots & & a_{6,6}
\end{array}\right) .
$$

In Eq. (7), the $6 \times 14$ order matrix $\boldsymbol{g}_{\alpha}$ is $\partial \boldsymbol{g} / \partial \boldsymbol{\alpha}$ and the $6 \times 1$ order matrix $g_{\mathrm{u}}$ is $\partial \boldsymbol{g} / \partial \boldsymbol{u}$, whose detail expressions are

$$
\boldsymbol{g}_{\alpha}=\left(\begin{array}{cccccc}
b_{1,1} & b_{1,2} & \cdots & b_{1,6} & \cdots & b_{1,14} \\
b_{2,1} & b_{2,2} & & & \vdots \\
\vdots & & \ddots & & \vdots \\
b_{6,1} & \cdots & \cdots & b_{6,6} & \cdots & b_{6,14}
\end{array}\right),
$$

and

$$
\boldsymbol{g}_{u}=\left(\begin{array}{c}
c_{1,1} \\
c_{2,1} \\
\vdots \\
c_{6,1}
\end{array}\right) .
$$

After Eqs. (13) and (14) are taken into Eq. (9), the PSM $S_{\alpha}$ can be expressed by $6 \times 14$ order matrix as follows:

$$
\boldsymbol{S}_{\alpha}=\left(\begin{array}{cccccc}
s_{1,1} & s_{1,2} & \cdots & s_{1,6} & \cdots & s_{1,14} \\
s_{1,2} & s_{2,2} & & & & \vdots \\
\vdots & & \ddots & & & \vdots \\
s_{6,1} & \cdots & \cdots & s_{6,6} & \cdots & s_{6,14}
\end{array}\right) .
$$

The $n$th row of the PSM $S_{\alpha}$ indicates the influence of seventeen parameters change on the $n$th state variable.

Similarly, after Eqs. (13) and (15) are taken into Eq. (9), the ISM $S_{\mathrm{u}}$ can be expressed by $6 \times 1$ order matrix as follows:

$$
\boldsymbol{S}_{\mathrm{u}}=\left(\begin{array}{c}
s_{1,1} \\
s_{2,1} \\
\vdots \\
s_{6,1}
\end{array}\right) \text {. }
$$

The $n$th row of the ISM $S_{\mathrm{u}}$ indicates the influence of one input change on the nth state variable.

The parameter vector $\Delta \boldsymbol{\alpha}$ is $14 \times 1$ order matrix, which can be expressed as follows:

$$
\Delta \boldsymbol{\alpha}=\left(\begin{array}{c}
\Delta \alpha_{1} \\
\Delta \alpha_{2} \\
\vdots \\
\Delta \alpha_{14}
\end{array}\right) .
$$

The input vector $\Delta \boldsymbol{u}$ is $1 \times 1$ order matrix, which can be expressed as follows:

$$
\Delta \boldsymbol{u}=\left(\Delta u_{1}\right) .
$$

After Eqs. (16)-(19) are taken into Eq. (11), the $6 \times 1$ order matrix $\Delta \boldsymbol{x}$ can be expressed as follows: 
Table 3 Nine working conditions selected by orthogonal test design

\begin{tabular}{lllc}
\hline No. & System pressure $\boldsymbol{P}_{\mathbf{s}}(\mathbf{M P a})$ & Frequency $\boldsymbol{f}(\mathbf{H z})$ & Amplitude $\boldsymbol{A}(\mathbf{N})$ \\
\hline 1 & 6 & 1 & 500 \\
2 & 6 & 2 & 1000 \\
3 & 6 & 4 & 1500 \\
4 & 12 & 1 & 1000 \\
5 & 12 & 2 & 1500 \\
6 & 12 & 4 & 500 \\
7 & 18 & 1 & 1500 \\
8 & 18 & 2 & 500 \\
9 & 18 & 4 & 1000 \\
\hline
\end{tabular}

$$
\begin{aligned}
\Delta \boldsymbol{x}=- & \left(\begin{array}{cccccc}
s_{1,1} & s_{1,2} & \cdots & s_{1,6} & \cdots & s_{1,14} \\
s_{1,2} & s_{2,2} & & & & \vdots \\
\vdots & & \ddots & & & \vdots \\
s_{6,1} & \cdots & \cdots & s_{6,6} & \cdots & s_{6,14}
\end{array}\right) \cdot\left(\begin{array}{c}
\Delta \alpha_{1} \\
\Delta \alpha_{2} \\
\vdots \\
\Delta \alpha_{14}
\end{array}\right)- \\
& \left(\begin{array}{c}
s_{1,1} \\
s_{2,1} \\
\vdots \\
s_{6,1}
\end{array}\right) \cdot\left(\Delta u_{1}\right)=\left(\begin{array}{c}
\Delta x_{1} \\
\Delta x_{2} \\
\vdots \\
\Delta x_{6}
\end{array}\right) .
\end{aligned}
$$

The $n$th row of the matrix $\Delta x$ indicates the sum influence of one input change and fourteen parameters change on the $n$th state variable.

When only the parameters change of HIVC rather than the input change are focused, Eq. (11) can be simplified as follows:

$$
\Delta x=-S_{\alpha} \cdot \Delta \alpha .
$$

The detail form of Eq. (21) can be expressed as follows:

$$
\begin{aligned}
\Delta \boldsymbol{x} & =-\left(\begin{array}{cccccc}
s_{1,1} & s_{1,2} & \cdots & s_{1,6} & \cdots & s_{1,14} \\
s_{1,2} & s_{2,2} & & & & \vdots \\
\vdots & & \ddots & & & \vdots \\
s_{6,1} & \cdots & \cdots & s_{6,6} & \cdots & s_{6,14}
\end{array}\right) \cdot\left(\begin{array}{c}
\Delta \alpha_{1} \\
\Delta \alpha_{2} \\
\vdots \\
\Delta \alpha_{14}
\end{array}\right) \\
& =\left(\begin{array}{c}
\Delta x_{1} \\
\Delta x_{2} \\
\vdots \\
\Delta x_{6}
\end{array}\right) .
\end{aligned}
$$

The input vector $\Delta Y$ is $1 \times 1$ order matrix, and the coefficient matrix $\boldsymbol{D}$ is zero matrix, so Eq. (11) can be simplified as follows:

$$
\Delta Y=C \cdot \Delta x=-C \cdot S_{\alpha} \cdot \Delta \alpha .
$$

Define

$$
\boldsymbol{S}_{\alpha}^{Y}=\boldsymbol{C} \cdot \boldsymbol{S}_{\alpha},
$$

where $\boldsymbol{S}_{\alpha}^{Y}$ is the PSM of output vector, $\boldsymbol{D}$ is coefficient matrix, $C$ is coefficient matrix.

Then, the output vector change can be expressed as follows:

$$
\Delta Y=-S_{\alpha}^{Y} \cdot \Delta \alpha
$$

Due to limited space, the detail expressions from Eqs. (13) to (25) are not listed in this paper.

\section{Sensitivity Analysis of HIVC 4.1 Dynamic Sensitivity Analysis 4.1.1 Orthogonal Test Design}

Sinusoidal response can evaluate the tracing result with different frequency and amplitude, and its control result indicates the response performance and the control accuracy, so the sinusoidal signal is chosen as the input signal to analyze the system parameter sensitivity of the HIVC force control system. Three working condition factors including the system supply oil pressure $P_{\mathrm{s}}$, the sinusoidal frequency $f$ and the sinusoidal amplitude $A$ are chosen and each factor has three levels. $P_{\mathrm{s}}$ are $6 \mathrm{MPa}, 12 \mathrm{MPa}$, and $18 \mathrm{MPa}, f$ are $1 \mathrm{~Hz}, 2 \mathrm{~Hz}$, and $4 \mathrm{~Hz}, A$ are $500 \mathrm{~N}$, $1000 \mathrm{~N}$ and $1500 \mathrm{~N}$. There should be $3^{3}=27$ working conditions which will need superfluous work to complete the comprehensive test.

The orthogonal test design can make the comprehensive and superfluous test simplified to a few tests and evaluate the comprehensive test $[25,26]$. The orthogonal test can be expressed as $L_{\mathrm{n}}\left(t^{c}\right)$, where $\mathrm{L}$ is the orthogonal test code, $\mathrm{n}$ is the test number and $\mathrm{c}$ is the number of the factors. In this paper, the orthogonal test of three factors and three levels can be expressed as $L_{9}\left(3^{4}\right)$, which needs nine tests to evaluate the comprehensive research, and the detail orthogonal test table is shown in Table 3.

The range analysis table of orthogonal test is needed to evaluate the results of the comprehensive test after the results of these nine selected working conditions shown in Table 3 are calculated. And the mean value of one level within one factor can be expressed as follows:

$$
k_{\beta}=\sum_{\gamma=1}^{l} k_{\beta \gamma},
$$

where $k_{\beta}$ is mean value of one factor and one level in range analysis, subscript $\beta$ shows the $\beta$ th level within one factor, subscript $\gamma$ shows the $\gamma$ th result within this level and $l$ shows the number of level. Therefore, $k_{\beta}$ can reflect the proportion of this level in all levels, and the larger value indicates that this level within one factor can cause greater influence on result. 


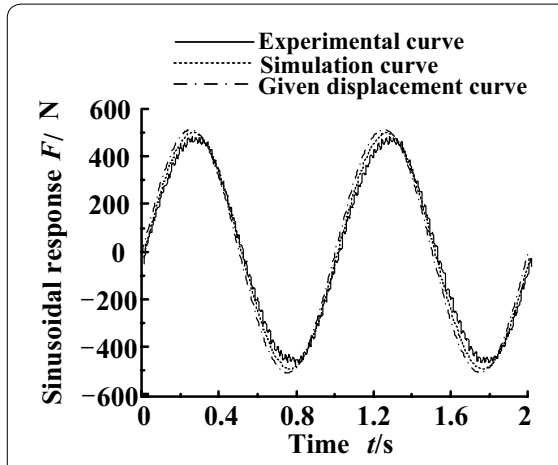

a $6 \mathrm{MPa}, 1 \mathrm{~Hz}, 500 \mathrm{~N}$

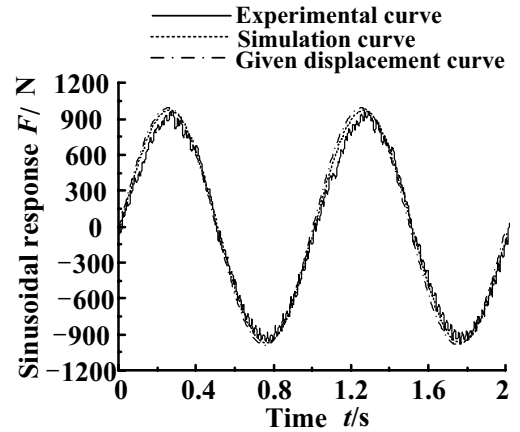

d $12 \mathrm{MPa}, 1 \mathrm{~Hz}, 1000 \mathrm{~N}$

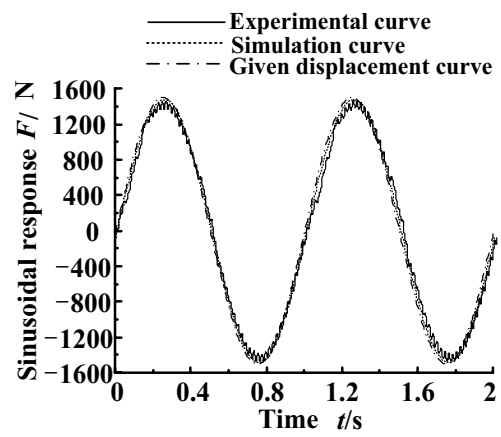

g $18 \mathrm{MPa}, 1 \mathrm{~Hz}, 1500 \mathrm{~N}$

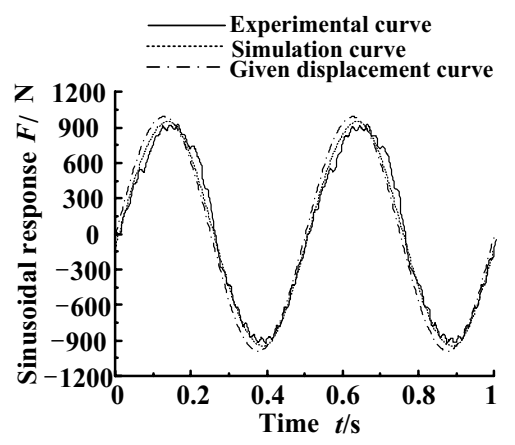

b $6 \mathrm{MPa}, 2 \mathrm{~Hz}, 1000 \mathrm{~N}$

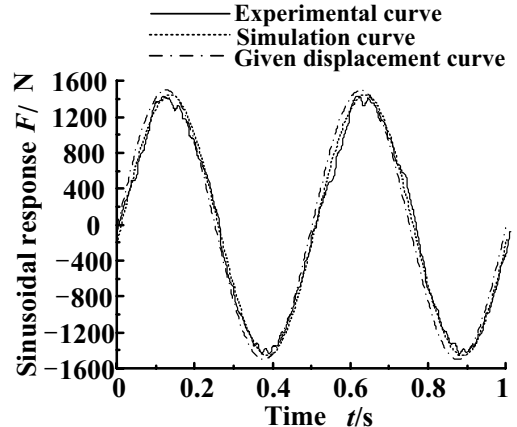

e $12 \mathrm{MPa}, 2 \mathrm{~Hz}, 1500 \mathrm{~N}$

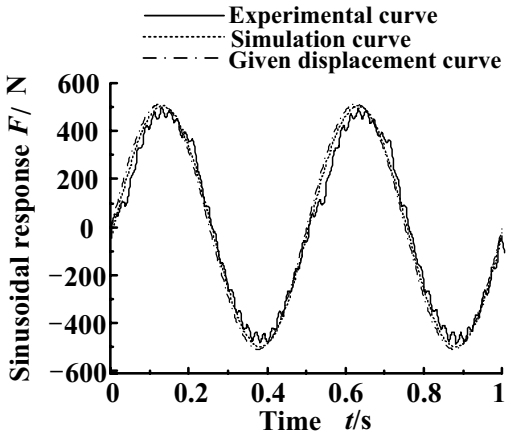

h $18 \mathrm{MPa}, 2 \mathrm{~Hz}, 500 \mathrm{~N}$

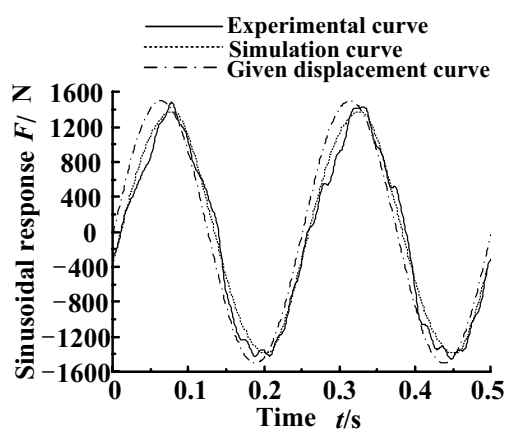

c $6 \mathrm{MPa}, 4 \mathrm{~Hz}, 1500 \mathrm{~N}$

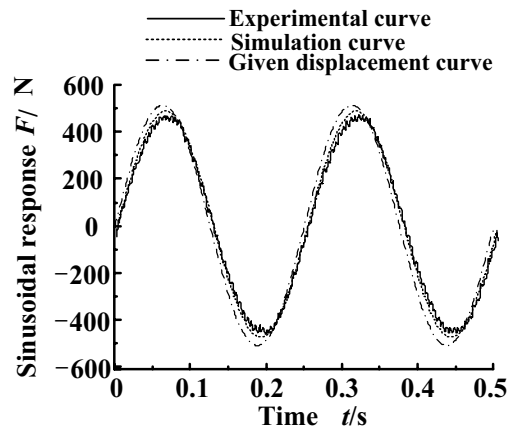

f $12 \mathrm{MPa}, 4 \mathrm{~Hz}, 500 \mathrm{~N}$

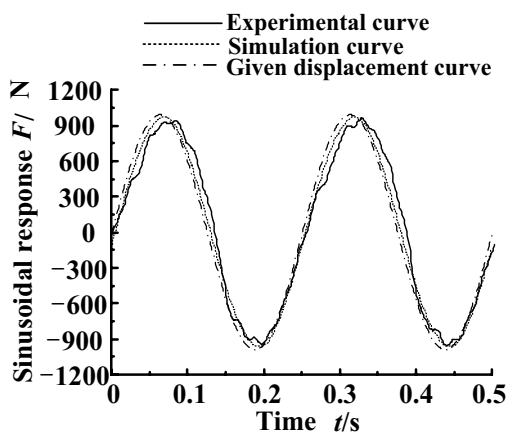

i $18 \mathrm{MPa}, 4 \mathrm{~Hz}, 1000 \mathrm{~N}$

Figure $\mathbf{5}$ Simulation and experimental curves of force sinusoidal response

And the change range of one factor can be expressed as follows:

$$
R=\left|\max \left(k_{\beta}\right)-\min \left(k_{\beta}\right)\right|,
$$

where $R$ is range of one factor in range analysis. $R$ can reflect the change range of each level within one factor, and the larger value can reflect that the levels change within one factor can cause greater influence on the result.

\subsubsection{Nine Working Conditions}

To indicate each system parameter sensitivity characteristics, the force sinusoidal response simulation and experiment curves with two stable periods under the nine selected working conditions are shown in Figure 5.

The amplitude attenuation and the phase lag are two typical performance indexes to evaluate the sinusoidal response and these two performance indexes under the nine selected working conditions are shown in Table 4 . 
Table 4 Performance indexes under the nine selected working conditions

\begin{tabular}{|c|c|c|c|c|c|c|c|c|c|}
\hline Performance index & 1 & 2 & 3 & 4 & 5 & 6 & 7 & 8 & 9 \\
\hline \multicolumn{10}{|c|}{ Amplitude attenuation $\Phi(\mathrm{N})$} \\
\hline Experiment & 32.1 & 76.5 & 101.5 & 31.3 & 40.1 & 30.4 & 29.2 & 18.8 & 38.2 \\
\hline Simulation & 9.5 & 41.7 & 185.9 & 12.1 & 31.2 & 18.6 & 14.2 & 6.1 & 27.1 \\
\hline \multicolumn{10}{|l|}{ Phase lag $\psi\left({ }^{\circ}\right)$} \\
\hline Experiment & 8.7 & 13.8 & 24.5 & 5.2 & 8.9 & 14.4 & 3.9 & 8.1 & 17.9 \\
\hline Simulation & 5.0 & 10.5 & 20.8 & 3.5 & 7.1 & 13.2 & 2.8 & 5.4 & 10.8 \\
\hline
\end{tabular}

As it can be seen in Table 4, the maximum amplitude attenuation difference between the simulation and the experiment is about $5.6 \%$, the maximum phase lag between the simulation and the experiment is $7.1^{\circ}$, and the maximum difference of the two performance indexes occurs when the sinusoidal frequency is $4 \mathrm{~Hz}$. As the frequency is smaller, the difference of the two performance indexes between the simulation and the experiment are smaller. Moreover, when the working condition is $6 \mathrm{MPa}$, $4 \mathrm{~Hz}$ and $1500 \mathrm{~N}$, the amplitude attenuation and phase lag differences between the simulation and the experiment are bigger than those under other working conditions, which is caused by that the maximum piston output force is about $1600 \mathrm{~N}$ when the system supply oil pressure is $6 \mathrm{MPa}$, and the bigger frequency can also lead the bigger values of the two performance indexes. Above all, the nine groups of simulation and experiment curves are fitting well respectively, which can indicate that the nonlinear mathematical model has relatively high accuracy and its parameters setting is accurate.

Based on the orthogonal test, the simulation and experiment results of the two performance indexes under the nine selected working conditions can be used to derive the range analysis, and the range analysis table is shown in Table 5, which can indicate the change rules of the two performance indexes under the whole working conditions.

As it can be seen in Table 5, the two performance indexes change rules of the simulation and experiment are the same. When the amplitude is bigger, the frequency is smaller or the supply oil pressure is smaller, the amplitude attenuation and the phase lag are bigger. Besides, the amplitude attenuation change range caused by these three factors change is similar, while the frequency change has the most effects on the phase lag and the amplitude change has the least effects on the phase lag.

\subsubsection{PSM}

For servo cylinder piston displacement, servo cylinder piston velocity, servo valve spool displacement, servo valve spool velocity and two servo cylinder cavities pressure are equal to 0 at the initial moment, the initial system state variable $x$ is $\boldsymbol{x}_{0}=\boldsymbol{0}$. And PSM $\boldsymbol{S}_{\alpha}$ are

$$
\boldsymbol{S}_{\alpha 0}=\mathbf{0}_{m \times p},
$$

where $\boldsymbol{O}_{m \times p}$ is $6 \times 14$ order zero matrix. And the PSM of output vector $\boldsymbol{S}_{\alpha}^{Y}$ can be expressed as follows:

$$
\boldsymbol{S}_{\alpha}^{Y}=A_{\mathrm{p}}\left(\boldsymbol{S}_{\alpha}^{5}-\boldsymbol{S}_{\alpha}^{6}\right),
$$

where the superscript 5 and 6 indicate the row number of PSM related to the state variables $x_{5}$ and $x_{6}$ respectively.

The associated equations including Eqs. (13), (14), (16), (18) and (29) are solved by programming on the MATLAB. Then the PSM $S_{\alpha}^{Y}$ of each system parameter are calculated under the nine selected working conditions, and the time-history curves of PSM $S_{\alpha}^{Y}$ under the first selected working condition which is $6 \mathrm{MPa}, 1 \mathrm{~Hz}, 500 \mathrm{~N}$ are shown in Figure 6.

As it can be seen in Figures 5(a) and 6, firstly, the PSM change rules of parameter $\alpha_{1}, \alpha_{5}, \alpha_{7}$ and $\alpha_{14}$ are the same. When the output force is zero as the piston speed is the largest, the PSM of parameter $\alpha_{1}, \alpha_{5}, \alpha_{7}$ and $\alpha_{14}$ are zero, and their change rules are sinusoidal. Secondly, the PSM of parameter $\alpha_{2}$ is also zero when the output force is zero, but its change rule is oppositely sinusoidal, which is different from those of parameter $\alpha_{1}, \alpha_{5}, \alpha_{7}$ and $\alpha_{14}$. Thirdly, the PSM change rules of parameter $\alpha_{4}, \alpha_{6}, \alpha_{8}$ and $\alpha_{10}$ are the same. When the output force is zero, the PSM of parameter $\alpha_{4}, \alpha_{6}$, $\alpha_{8}$ and $\alpha_{10}$ are the biggest and their change rules are cosinoidal. Fourthly, The PSM change rules of the other parameter $\alpha_{3}, \alpha_{9}, \alpha_{11}, \alpha_{12}$ and $\alpha_{13}$ are the same. When the output force is zero, the PSM of parameter $\alpha_{3}, \alpha_{9}, \alpha_{11}, \alpha_{12}$ and $\alpha_{13}$ are the biggest and their change rules are oppositely cosinoidal.

Due to limited space, the PSM of the other eight working conditions are not listed in this paper.

\subsubsection{Time-history Comparative Curves of Force Change}

In order to indicate the influence of each parameter change on the sinusoidal force response characteristics of HIVC under the nine selected working conditions clearly, the force output change $\Delta Y$ can be expressed as 
Table 5 Range analysis table of two performance indexes

\begin{tabular}{|c|c|c|c|c|c|c|c|c|c|c|c|c|}
\hline \multirow[t]{2}{*}{ Performance indexes } & \multicolumn{6}{|c|}{ Amplitude attenuation $\Phi(\mathrm{N})$} & \multicolumn{6}{|c|}{ Phase lag $\psi\left(10^{-2}\left(^{\circ}\right)\right)$} \\
\hline & \multicolumn{2}{|l|}{$P_{\mathrm{s}}$} & \multicolumn{2}{|l|}{$f$} & \multicolumn{2}{|l|}{$A$} & \multicolumn{2}{|l|}{$P_{\mathrm{s}}$} & \multicolumn{2}{|l|}{$f$} & \multicolumn{2}{|l|}{$A$} \\
\hline Factors & Exp. & Sim. & Exp. & Sim. & Exp. & Sim. & Exp. & Sim. & Exp. & Sim. & Exp. & Sim. \\
\hline$k_{1}$ & 70.0 & 79.0 & 30.9 & 11.9 & 27.1 & 11.4 & 15.7 & 12.1 & 5.9 & 3.8 & 10.4 & 7.9 \\
\hline$k_{2}$ & 33.9 & 20.6 & 45.1 & 26.3 & 48.7 & 27.0 & 9.5 & 7.9 & 10.3 & 7.7 & 12.3 & 8.3 \\
\hline$k_{3}$ & 28.7 & 15.8 & 56.7 & 77.2 & 56.9 & 77.1 & 10.0 & 6.3 & 18.9 & 14.9 & 12.4 & 10.2 \\
\hline$R$ & 41.3 & 63.2 & 25.8 & 65.3 & 29.8 & 65.7 & 6.2 & 5.8 & 13.0 & 11.1 & 2.0 & 2.3 \\
\hline
\end{tabular}

$$
\Delta Y=-S_{\alpha}^{Y} \cdot \Delta \alpha_{\mathrm{i}}
$$

According to Eq. (30), the time-history curves of $\Delta Y$ are shown in Figure 7, based on the PSM $\boldsymbol{S}_{\alpha}^{Y}$ with parameter increase $10 \%$ under the nine selected working conditions.

As it can be seen in Figure 7, the system parameters increment can cause the output force change periodically. In the following section, these dynamic changes are quantitatively analyzed to grasp the sensitivity change rule of each parameter under the nine selected working conditions.

\subsection{Quantitative Sensitivity Analysis \\ 4.2.1 Sensitivity Indexes}

In this section, two measurable indexes of parameters sensitivity are defined to quantitatively analyze the characteristics of force change caused by the parameters change under the nine selected working conditions.

Denote the first measurable index of parameters sensitivity $s_{1}$ which is the mean valve of the amplitude attenuation change after the parameters increase $10 \%$ in one stable period as follows:

$$
s_{1}=\operatorname{mean}\left(\Phi_{1}+\Phi_{2}\right),
$$

where

$$
\begin{aligned}
\Phi_{1} & =\left[\max \left(F_{\mathrm{r}}\right)-\max \left(F-S_{\alpha}^{Y} \cdot \Delta \alpha_{\mathrm{i}}\right)\right]-\left[\max \left(F_{\mathrm{r}}\right)-\max (F)\right] \\
& =\max (F)-\max \left(F-S_{\alpha}^{Y} \cdot \Delta \alpha_{\mathrm{i}}\right),
\end{aligned}
$$

$$
\begin{aligned}
\Phi_{2} & =\left[\min \left(F_{\mathrm{r}}\right)-\min (F)\right]-\left[\min \left(F_{\mathrm{r}}\right)-\min \left(F-S_{\alpha}^{Y} \cdot \Delta \alpha_{\mathrm{i}}\right)\right] \\
& =\min \left(F-S_{\alpha}^{Y} \cdot \Delta \alpha_{\mathrm{i}}\right)-\min (F),
\end{aligned}
$$

where $\Phi_{1}$ is amplitude attenuation change caused by the parameters change at the peak in one stable period, $\Phi_{2}$ is amplitude attenuation change caused by the parameters change at the trough in one stable period. Similarly,denote the second measurable index of parameters sensitivity $s_{2}$ which is the mean valve of the phase lag change after the parameters increase $10 \%$ in one stable period as follows:

$$
s_{2}=\operatorname{mean}(\Psi),
$$

where $\psi$ is phase lag change caused by the parameters change in one stable period, as follows:

$$
\begin{aligned}
\Psi= & {\left[\arcsin \left(\frac{F_{\mathrm{r}}}{\max \left(F_{\mathrm{r}}\right)}\right)-\arcsin \left(\frac{F-\mathbf{S}_{\alpha}^{Y} \cdot \Delta \alpha_{\mathrm{i}}}{\max \left(F-\mathbf{S}_{\alpha}^{Y} \cdot \Delta \alpha_{\mathrm{i}}\right)}\right)\right] } \\
& -\left[\arcsin \left(\frac{F_{\mathrm{r}}}{\max \left(F_{\mathrm{r}}\right)}\right)-\arcsin \left(\frac{F}{\max (F)}\right)\right] .
\end{aligned}
$$

The influence degree of parameters change on piston output force $F$ can be evaluated quantitatively and clearly by analyzing these two sensitivity indexes.

\subsubsection{Sensitivity Indexes Histograms}

According to Eqs. (31) and (34), the two sensitivity indexes histograms are obtained to illustrate the sensitivity characteristics of each system parameter with each parameter increase $10 \%$ under the nine selected working conditions. The histograms are shown in Figure 8.

As it can be seen in Figure 8, the sensitivity characteristics of each parameter in HIVC under the nine selected working conditions are as below.

(1) The effects of each parameter increase $10 \%$ on the amplitude attenuation and phase lag are different under the same working condition. The two sensitivity indexes of parameters $\alpha_{4}, \alpha_{6}, \alpha_{7}, \alpha_{9}$ and $\alpha_{10}$ are quite smaller than those of the other parameters under the nine selected working conditions, indicating that the small fluctuation of the five parameters has few effects on the HIVC force control performance. To research the main performanceinfluenced parameters selectively, the sensitivity of parameters $\alpha_{4}, \alpha_{6}, \alpha_{7}, \alpha_{9}$ and $\alpha_{10}$ is not detailedly researched in this paper. And both $\alpha_{11}$ and $\alpha_{12}$ are the forward path gains which have completely same sensitivity characteristics, so only the sensitivity characteristics of parameter $\alpha_{12}$ are analyzed in the following sections. The effects of parameters $\alpha_{1}$, 

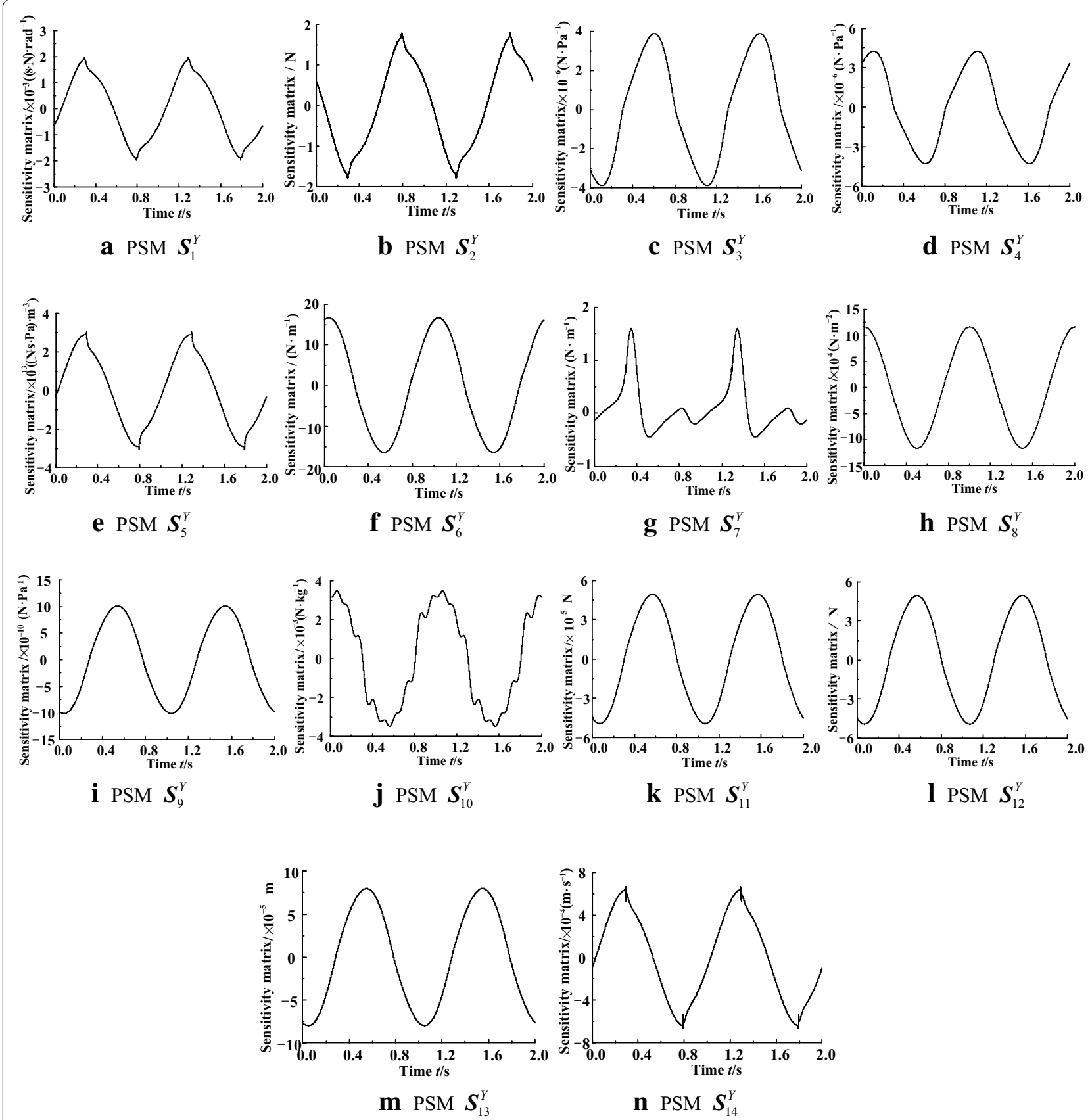

Figure 6 PSM curves with $6 \mathrm{MPa}, 1 \mathrm{~Hz}, 500 \mathrm{~N}$

$\alpha_{2}, \alpha_{3}, \alpha_{5}, \alpha_{8}, \alpha_{12}, \alpha_{13}$ and $\alpha_{14}$ increase $10 \%$ on the amplitude attenuation and phase lag are large and their sensitivity characteristics must be analyzed detailedly in the following.

(2) The parameters increase has positive or negative effects on the amplitude attenuation and phase lag. Taking sensitivity characteristics of $\alpha_{3}, \alpha_{12}$ and $\alpha_{13}$ as examples, the increasing of them can decrease the amplitude attenuation and phase lag, which is caused by that the increasing of the system supply oil pressure $\alpha_{3}$ and control gain $\alpha_{12}$ will increase the channel gain of the transfer function to enhance the system response speed and control accuracy. According to Figure 8, the parameters having positive/negative effects on the system control performance should be enlarged/restrained to optimize 


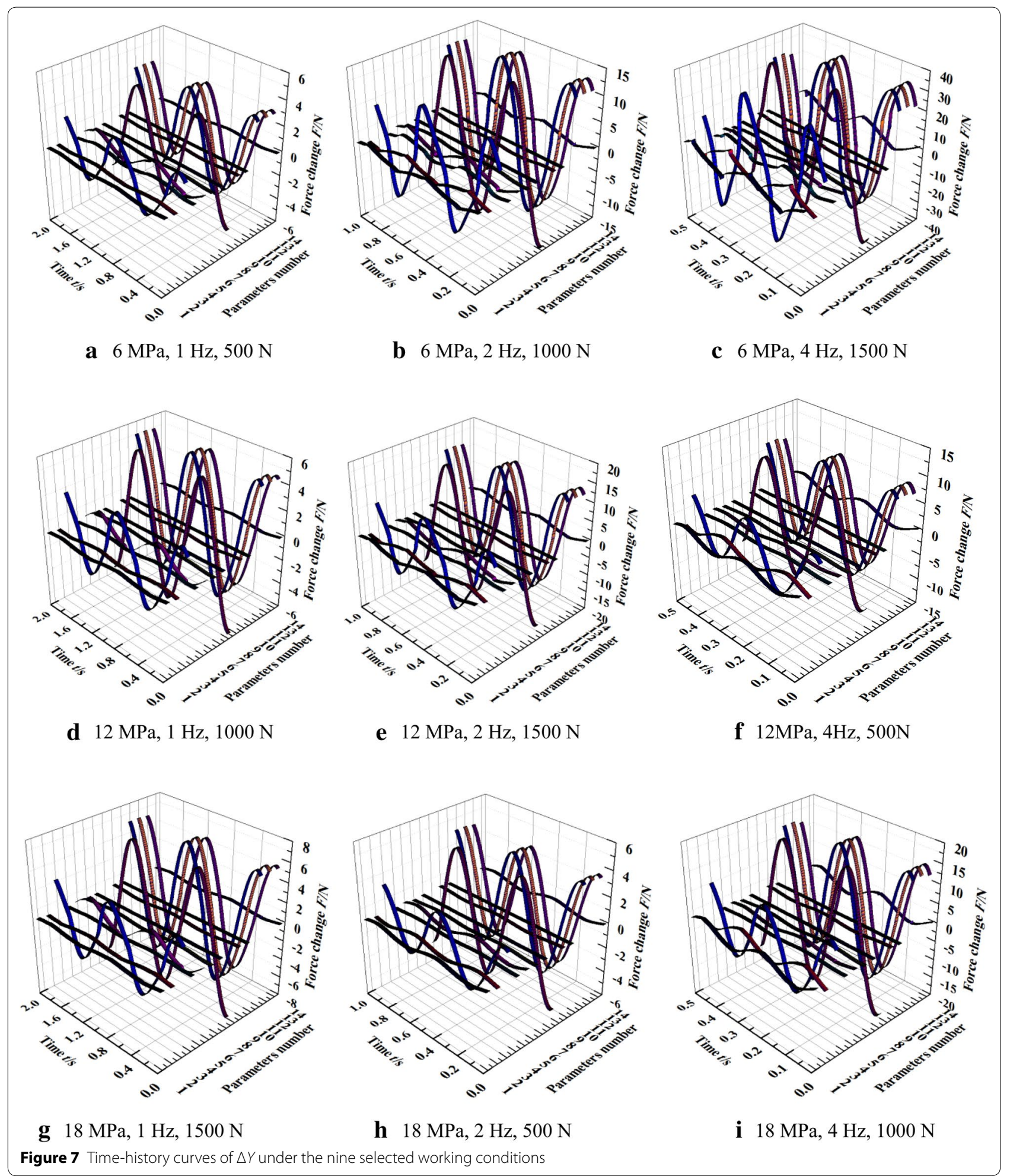




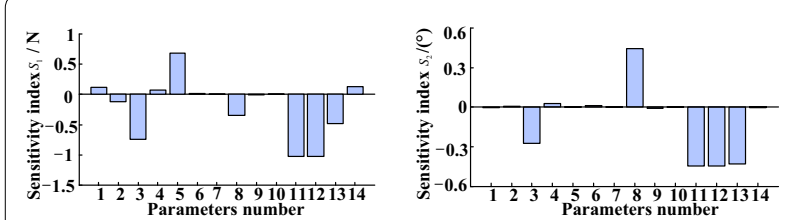

a $s_{1}$ and $s_{2}$ with $6 \mathrm{MPa}, 1 \mathrm{~Hz}, 500 \mathrm{~N}$
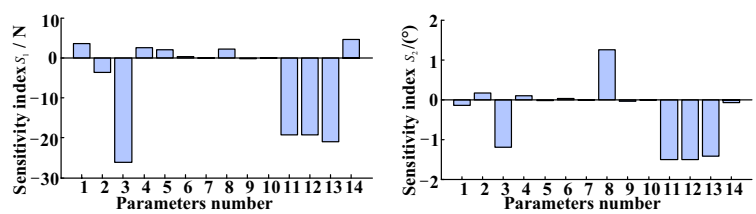

c $s_{1}$ and $s_{2}$ with $6 \mathrm{MPa}, 4 \mathrm{~Hz}, 1500 \mathrm{~N}$
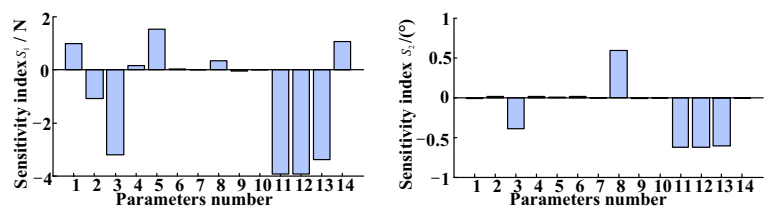

e $s_{1}$ and $s_{2}$ with $12 \mathrm{MPa}, 2 \mathrm{~Hz}, 1500 \mathrm{~N}$
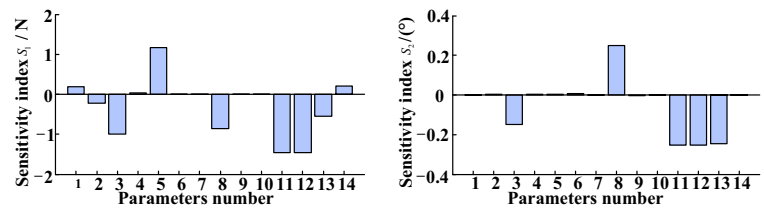

g $s_{1}$ and $s_{2}$ with $18 \mathrm{MPa}, 1 \mathrm{~Hz}, 1500 \mathrm{~N}$
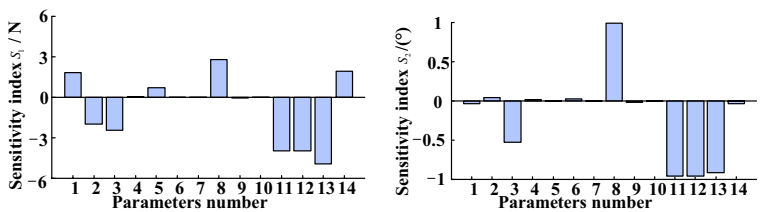

i $s_{1}$ and $s_{2}$ with $18 \mathrm{MPa}, 4 \mathrm{~Hz}, 1000 \mathrm{~N}$
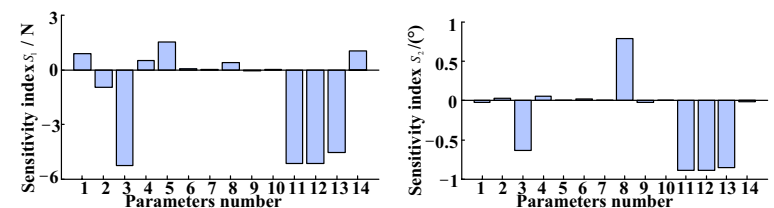

b $s_{1}$ and $s_{2}$ with $6 \mathrm{MPa}, 2 \mathrm{~Hz}, 1000 \mathrm{~N}$
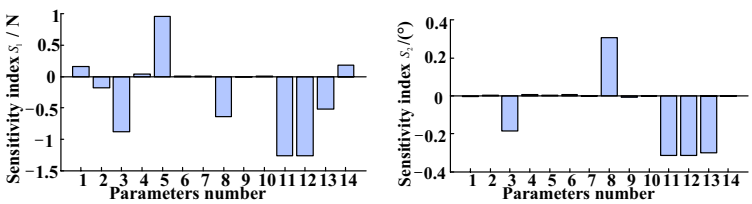

d $s_{1}$ and $s_{2}$ with $12 \mathrm{MPa}, 1 \mathrm{~Hz}, 1000 \mathrm{~N}$
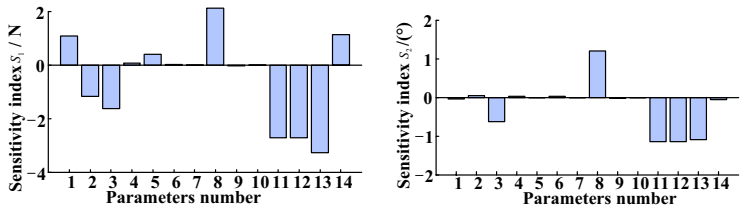

f $s_{1}$ and $s_{2}$ with $12 \mathrm{MPa}, 4 \mathrm{~Hz}, 500 \mathrm{~N}$

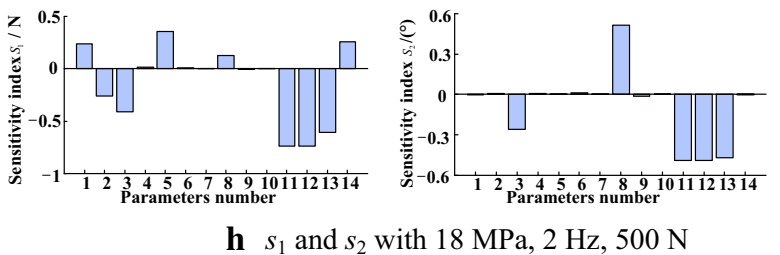

h $s_{1}$ and $s_{2}$ with $18 \mathrm{MPa}, 2 \mathrm{~Hz}, 500 \mathrm{~N}$

Figure 8 Sensitivity indexes histograms under the selected nice working conditions

the structure design and control strategy, which can improve the whole force control performance of HIVC. On the contrary, the secondary performance-influenced parameter whose fluctuation is small can be ignored.

(3) The amplitude attenuation and the phase lag can be used to evaluate the force control accuracy and response speed respectively, yet the two sensitivity indexes plus-minus characteristics of some parameters are not the same. The two sensitivity indexes plus-minus characteristics of $\alpha_{1}, \alpha_{5}$ and $\alpha_{14}$ is opposite, when these three parameters increase, the amplitude attenuation increases and the phase lag decreases. The two performance indexes plusminus characteristics of $\alpha_{8}$ is opposite under some working conditions but the same under the other working conditions. Qualitatively speaking, the effective piston area of servo cylinder increasing enlarges the output force, which improves the system performance based on the force equilibrium equation, but the effective piston area of servo cyl- 
Table 6 Range analysis of each parameter $S_{1}(\mathrm{~N})$

\begin{tabular}{|c|c|c|c|c|c|c|c|c|c|c|c|c|}
\hline \multirow{2}{*}{$\begin{array}{l}\text { Parameters } \\
\text { Factors }\end{array}$} & \multicolumn{3}{|l|}{$\omega$} & \multicolumn{3}{|l|}{$\zeta$} & \multicolumn{3}{|l|}{$P_{s}$} & \multicolumn{3}{|l|}{$C_{\text {ip }}$} \\
\hline & $P_{\mathrm{s}}$ & $f$ & $A$ & $P_{\mathrm{s}}$ & $f$ & $A$ & $P_{\mathrm{s}}$ & $f$ & $A$ & $P_{s}$ & $f$ & $A$ \\
\hline$k_{1}$ & 1.5 & 0.2 & 0.5 & -1.6 & -0.2 & -0.5 & -10.7 & -0.9 & -0.9 & 1.4 & 1.0 & 0.5 \\
\hline$k_{2}$ & 0.8 & 0.7 & 1.0 & -0.8 & -0.8 & -1.1 & -1.9 & -3.0 & -2.9 & 1.0 & 1.1 & 1.0 \\
\hline$k_{3}$ & 0.8 & 2.2 & 1.6 & -0.8 & -2.2 & -1.6 & -1.3 & -10.1 & -10.1 & 0.8 & 1.0 & 1.6 \\
\hline$R$ & 0.7 & 2.0 & 1.1 & 0.8 & 2.0 & 1.1 & 9.4 & 9.2 & 9.2 & 0.6 & 0.1 & 1.1 \\
\hline Parameters & \multicolumn{3}{|l|}{$A_{\mathrm{p}}$} & \multicolumn{3}{|l|}{$K_{p}$} & \multicolumn{3}{|l|}{$K$} & \multicolumn{3}{|l|}{$B_{p}$} \\
\hline Factors & $P_{\mathrm{s}}$ & $f$ & $A$ & $P_{\mathrm{s}}$ & $f$ & $A$ & $P_{\mathrm{s}}$ & $f$ & $A$ & $P_{\mathrm{s}}$ & $f$ & $A$ \\
\hline$k_{1}$ & 0.7 & -0.6 & 0.6 & -8.5 & -1.3 & -1.5 & -8.7 & -0.5 & -1.5 & 1.9 & 0.2 & 0.5 \\
\hline$k_{2}$ & 0.6 & 0.3 & 0.9 & -2.6 & -3.3 & -3.5 & -2.4 & -2.8 & -3.3 & 0.8 & 0.8 & 1.1 \\
\hline$k_{3}$ & 0.6 & 2.4 & 0.5 & -2.1 & -8.6 & -8.2 & -2 & -9.7 & -8.3 & 0.8 & 2.5 & 1.9 \\
\hline$R$ & 0.1 & 3.0 & 0.4 & 6.4 & 7.3 & 6.7 & 6.7 & 9.2 & 6.8 & 1.1 & 2.3 & 1.4 \\
\hline
\end{tabular}

Table 7 Range analysis of each parameter $S_{2}\left(10^{-2}\left({ }^{\circ}\right)\right)$

\begin{tabular}{|c|c|c|c|c|c|c|c|c|c|c|c|c|c|}
\hline \multirow{2}{*}{$\begin{array}{l}\text { Parameters } \\
\text { Factors }\end{array}$} & \multicolumn{4}{|l|}{$\omega$} & \multicolumn{3}{|l|}{$\zeta$} & \multicolumn{3}{|l|}{$P_{s}$} & \multicolumn{3}{|l|}{$C_{i p}$} \\
\hline & $P_{\mathrm{s}}$ & $f$ & $A$ & $A$ & $P_{\mathrm{s}}$ & $f$ & $A$ & $P_{\mathrm{s}}$ & $f$ & $A$ & $P_{\mathrm{s}}$ & $f$ & $A$ \\
\hline$k_{1}$ & -5.5 & -0.2 & & -1.6 & 6.8 & 0.3 & 4.5 & -70.2 & -20.2 & -38.8 & -0.4 & 0.2 & 0.1 \\
\hline$k_{2}$ & -1.8 & -1.2 & & -1.8 & 2.5 & 3.8 & 2.5 & -40.2 & -43.0 & -44.9 & 0.1 & 0.1 & 0.1 \\
\hline$k_{3}$ & -1.2 & -7.0 & & -5.0 & 4.0 & 9.2 & 6.2 & -31.2 & -78.3 & -57.9 & 0.1 & -0.6 & -0.4 \\
\hline$R$ & 4.3 & 6.8 & & 3.4 & 4.3 & 8.9 & 3.7 & 39.0 & 58.1 & 19.1 & 0.5 & 0.8 & 0.5 \\
\hline Parameters & \multicolumn{4}{|l|}{$A_{\mathrm{p}}$} & \multicolumn{3}{|l|}{$K_{\mathrm{p}}$} & \multicolumn{3}{|l|}{$K$} & \multicolumn{3}{|l|}{$B_{p}$} \\
\hline Factors & $P_{\mathrm{s}}$ & $f$ & $A$ & & $P_{s}$ & $f$ & $A$ & $P_{\mathrm{s}}$ & $f$ & $A$ & $P_{\mathrm{s}}$ & $f$ & $A$ \\
\hline$k_{1}$ & 83.3 & 33.4 & 72.1 & & -94.5 & -33.8 & -69.5 & -90.1 & -32.6 & -66.5 & -2.9 & -0.1 & -1.7 \\
\hline$k_{2}$ & 70.2 & 63.6 & 69.8 & & -69.5 & -66.8 & -71.9 & -66.5 & -64.5 & -69.0 & -1.6 & -0.5 & -1.3 \\
\hline$k_{3}$ & 58.5 & 115.0 & 70.2 & & -56.6 & -120.1 & -79.3 & -54.3 & -113.8 & -75.4 & -1.2 & -5 & -2.6 \\
\hline$R$ & 24.8 & 81.6 & 2.3 & & 37.9 & 86.3 & 9.8 & 35.8 & 81.2 & 8.9 & 1.7 & 4.9 & 1.3 \\
\hline
\end{tabular}

inder increasing enlarges the compressible oil volume of the servo cylinder, which delays the pressure building time and has negative effects on the system performance. So the effect of it on the system performance is strongly related to the working conditions.

(4) As the working condition changes, the two sensitivity indexes proportions of one parameter change, compared with those of the other parameters. The two sensitivity indexes proportions of $\alpha_{1}, \alpha_{3}, \alpha_{5}, \alpha_{8}$, and $\alpha_{14}$ are large under some working conditions but small under the other working condition, the compensation control are conducted to improve the HIVC force control performance.

(5) The two sensitivity indexes magnitude of different parameters is related to the magnitude of the amplitude attenuation and phase lag directly under one working condition. In other words, when the magnitude of the amplitude attenuation and the phase lag is large or small, the magnitude of $S_{1}$ and $S_{2}$ is large or small.

\subsubsection{Range Analysis}

In this section, according to the two sensitivity indexes histogram under the nine selected working conditions shown in Figure 8, the two sensitivity indexes range analysis tables based on the orthogonal test theory are shown in Table 6 and Table 7 respectively which can indicate the change rules of sensitivity indexes to analyze the effects of each parameter on force control performance quantitatively and the differences under different working conditions. 

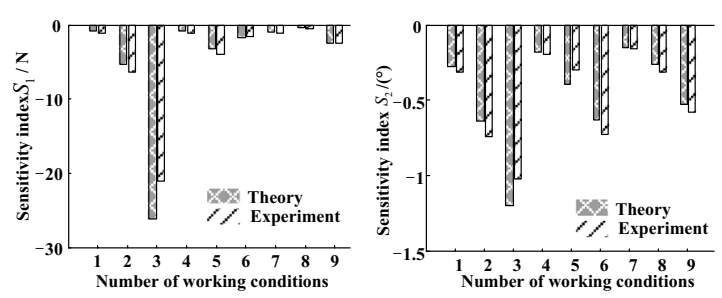

a System pressure $P_{\mathrm{s}}$

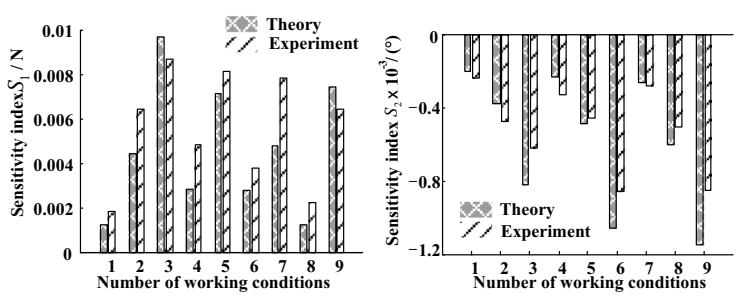

b Initial piston position of servo cylinder $L_{0}$
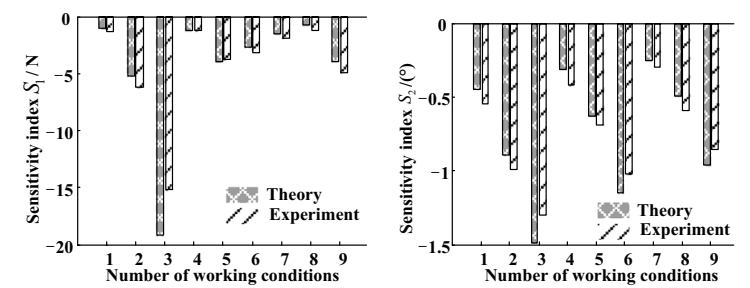

c Proportional gain $K_{p}$

Figure 9 Sensitivity index comparative histograms with parameter increase 10\%

As it can be seen in Table 6 and Table 7, the sensitivity change rules of each parameter in HIVC under the whole working conditions are below:

Firstly, the mean value of one level within one factor $k_{\beta}$ can indicate that the change rules of the effects that each factor has on the two sensitivity indexes of parameters $\alpha_{1}, \alpha_{2}, \alpha_{3}, \alpha_{12}, \alpha_{13}$ and $\alpha_{14}$ are quite similar to the change rules of the amplitude attenuation and phase lag shown in Table 5 under the whole working conditions. Frequency $f$ has small effects on $S_{1}$ of $\alpha_{5}$, and each factor change has effects on the plus-minus characteristic of its $S_{2}$. In addition, the effects of each parameter on $S_{1}$ of $\alpha_{8}$ are different from those of the other parameters, and even the plus-minus characteristics of its $S_{1}$ can be changed with the frequency $f$ change, yet the frequency $f$ has few effects on its $S_{2}$.

Secondly, the change range of one factor $R$ can indicate that the frequency $f$ is the main factor influencing $S_{1}$ of $\alpha_{1}, \alpha_{2}, \alpha_{8}, \alpha_{13}$ and $\alpha_{14}$. And the amplitude $A$ is the main factor influencing $S_{1}$ of $\alpha_{5}$. Moreover, each factor of $\alpha_{3}$ and $\alpha_{11}$ has similar effects on $S_{1}$. While the frequency $f$ is the main factor influencing $S_{2}$ of all the parameters, which can indicate that frequency $f$ has great effects on the phase lag.

\section{Experiments}

Due to the limited experimental conditions and the immeasurable parameters, lots of parameters in force control system of HIVC can't be measured directly so that their sensitivity characteristics can't be verified experimentally. However, all parameters sensitivity analysis is based on the same force control mathematical model and the sensitivity mathematical model, the analogy method can be used to deduce some immeasurable parameters sensitivity characteristics from experimental verification of some measurable parameters sensitivity characteristics.

System supply oil pressure $\alpha_{3}$, initial piston position of servo cylinder $\alpha_{7}$ and proportional gain $\alpha_{12}$ can be measured online, so their sensitivity characteristics can be verified experimentally. The initial output force is subtracted from the final output force caused by the parameters increasing. Moreover, the multiple-sampling average method is adopted to ensure the experimental results accuracy. And the comparative histograms of two sensitivity indexes with parameters increase 10\% under the nine selected working conditions are shown from Figure 9.

As it can be seen in Figure 9, the two sensitivity indexes experimental results of $L_{0}$ are quite small, which can verify the theoretical results of them. Therefore, the effects of $L_{0}$ on the force control performance can be ignored when it has relatively small fluctuation, and the detail results of it are not analyzed in this section. Moreover, the two sensitivity indexes experimental results of $P_{s}$ and $K_{p}$ are quite close to the theoretical results, and the detail range and error analysis of their sensitivity indexes under the whole working conditions are shown in Table 8 and Table 9, in which errors of $S_{1}$ and $S_{2}$ are both equal to the absolute value of the difference between the experiment and the simulation values.

As it can be seen in Table 8 and Table 9, the two sensitivity indexes differences of $P_{s}$ and $K_{p}$ between experimental results and theoretical results are small under the whole working conditions. For $P_{s}$, its $S_{1}$ maximum differences of $k_{\beta}$ and $R$ are 1.7 and 1.9, respectively, and its $S_{2}$ maximum differences of $k_{\beta}$ and $R$ are $0.092^{\circ}$ and $0.121^{\circ}$, respectively. For $K_{p}$, its $S_{1}$ maximum differences of $k_{\beta}$ and $R$ are 1.2 and 1.6, respectively, and its $S_{2}$ maximum differences of $k_{\beta}$ and $R$ are $0.092^{\circ}$ and $0.121^{\circ}$, respectively. Furthermore, the large differences are usually generated when the $P_{s}$ is small and the $f$ and $A$ are large.

The reason why the differences exist is that the force control system mathematical model of HIVC whose accuracy influences each system parameter sensitivity 
Table 8 Range and error analysis table of $\boldsymbol{P}_{\mathrm{s}}$ sensitivity indexes

\begin{tabular}{|c|c|c|c|c|c|c|c|c|c|c|c|c|c|c|c|c|c|c|}
\hline \multirow{3}{*}{$\begin{array}{l}\text { Sensitivity indexes } \\
\text { Factors }\end{array}$} & \multicolumn{9}{|l|}{$S_{1}(\mathrm{~N})$} & \multicolumn{9}{|c|}{$S_{2}\left(10^{-2}\left({ }^{\circ}\right)\right)$} \\
\hline & \multicolumn{3}{|l|}{$P_{s}$} & \multicolumn{3}{|l|}{$f$} & \multicolumn{3}{|l|}{$A$} & \multicolumn{3}{|l|}{$P_{s}$} & \multicolumn{3}{|l|}{$f$} & \multicolumn{3}{|l|}{$A$} \\
\hline & Exp. & Sim. & Err. & Exp. & Sim. & Err. & Exp. & Sim. & Err. & Exp. & Sim. & Err. & Exp. & Sim. & Err. & Exp. & Sim. & Err. \\
\hline$k_{1}$ & -9.5 & -10.7 & 1.2 & -1.1 & -0.9 & 0.2 & -1.0 & -0.9 & 0.1 & -66.3 & -70.2 & 3.9 & -21.2 & -20.2 & 1.0 & -41.0 & -38.8 & 3.2 \\
\hline$k_{2}$ & -2.2 & -1.9 & 0.3 & -3.5 & -3.0 & 0.5 & -3.2 & -2.9 & 0.3 & -40.8 & -40.2 & 0.6 & -44.1 & -43.0 & 1.1 & -50.3 & -44.9 & 5.4 \\
\hline$k_{3}$ & -1.3 & -1.3 & 0 & -8.4 & -10.1 & 1.7 & -8.7 & -10.1 & 1.4 & -34.5 & -31.2 & 3.3 & -76.5 & -78.3 & 1.8 & -48.7 & -57.9 & 9.2 \\
\hline$R$ & 8.2 & 9.4 & 1.2 & 7.3 & 9.2 & 1.9 & 7.7 & 9.2 & 1.5 & 31.8 & 39.0 & 7.2 & 55.3 & 58.1 & 2.8 & 7.7 & 19.1 & 12.1 \\
\hline
\end{tabular}

Table 9 Range analysis table of $K_{p}$ sensitivity indexes

\begin{tabular}{|c|c|c|c|c|c|c|c|c|c|c|c|c|c|c|c|c|c|c|}
\hline \multirow{3}{*}{$\begin{array}{l}\text { Sensitivity indexes } \\
\text { Factors }\end{array}$} & \multicolumn{9}{|l|}{$S_{1}(\mathrm{~N})$} & \multicolumn{9}{|c|}{$S_{2}\left(10^{-2}\left({ }^{\circ}\right)\right)$} \\
\hline & \multicolumn{3}{|l|}{$P_{\mathrm{s}}$} & \multicolumn{3}{|l|}{$f$} & \multicolumn{3}{|l|}{$A$} & \multicolumn{3}{|l|}{$P_{\mathrm{s}}$} & \multicolumn{3}{|l|}{$f$} & \multicolumn{3}{|l|}{$A$} \\
\hline & Exp. & Sim. & Err. & Exp. & Sim. & Err. & Exp. & Sim. & Err. & Exp. & Sim. & Err. & Exp. & Sim. & Err. & Exp. & Sim. & Err. \\
\hline$k_{1}$ & -7.5 & -8.5 & 1.0 & -1.5 & -1.3 & 0.2 & -1.9 & -1.5 & 0.4 & -92.1 & -94.5 & 2.4 & -41.7 & -33.8 & 7.9 & -71.8 & -69.5 & 2.3 \\
\hline$k_{2}$ & -2.7 & -2.6 & 0.1 & -3.7 & -3.3 & 0.4 & -4.0 & -3.5 & 0.5 & -70.5 & -69.5 & 1.0 & -75.4 & -66.8 & 8.6 & -74.8 & -71.9 & 2.9 \\
\hline$k_{3}$ & -2.6 & -2.1 & 0.5 & -7.8 & -8.6 & 0.8 & -7.0 & -8.2 & 1.2 & -57.9 & -56.6 & 1.3 & -105.8 & -120.1 & 14.3 & -75.4 & -79.3 & 3.9 \\
\hline$R$ & 4.9 & 6.4 & 1.5 & 6.3 & 7.3 & 1.0 & 5.1 & 6.7 & 1.6 & 34.2 & 37.9 & 3.7 & 64.1 & 86.3 & 22.2 & 3.6 & 9.8 & 6.2 \\
\hline
\end{tabular}

analysis results can't accurately describe all the practical system characteristics, so the differences between the simulation results and experiment results are inevitable.

\section{Conclusions}

(1) The PSM of each parameter and the dynamic figures of the output force change with each parameter increase $10 \%$ can indicate that each parameter change has different dynamic effects on the sinusoidal output force and each parameter PSM changes periodically including sinusoid, opposite sinusoid, cosine and opposite cosine whose ranges are different and change with different working conditions.

(2) The two sensitivity indexes are built to evaluate the effects of each parameter increase $10 \%$ on the amplitude attenuation and the phase lag of the sinusoidal output force response. The results can indicate that system parameters increasing have positive or negative effects on the two sensitivity indexes. Moreover, the range analysis can indicate that the two sensitivity indexes of each parameter change with different working conditions including three working conditions factor.

(3) The two sensitivity indexes of the system return oil pressure, the total piston stroke of servo cylinder,the initial piston position of servo cylinder, the effective bulk modulus and the conversion mass are small under the nine selected working conditions. While the two sensitivity indexes of the natural frequency of servo valve, the damping ratio of servo valve, the system supply oil pressure, the internal leakage coefficient, the effective piston area of servo cylinder, the proportional gain, the force sensor gain and the viscous damping coefficient are large.

Authors' Contributions

$X-D K$ and L-JK were in charge of the whole trial; K-XB and BY wrote the manuscript; H-LZ, Q-XZ and C-HL assisted with sampling and laboratory analyses. All authors read and approved the final manuscript.

\section{Author details}

1 School of Mechanical Engineering, Yanshan University, Qinhuangdao 066004 China. ${ }^{2}$ State Key Laboratory of Fluid Power and Mechatronic System, Zhejiang University, Hangzhou 310027, China. ${ }^{3}$ Columbia University, New York 10027, USA.

\section{Authors' Information}

Kai-Xian Ba, born in 1989, is currently a PhD candidate at Yanshan University, China. His research interest is electro-hydraulic servo control system. Tel: +8618630472204; E-mail: bkx@ysu.edu.cn.

Bin Yu, born in 1985, is currently a lecture at Yanshan University, China. His research interest is fluid transmission and robot control. Tel: +8613930342639; E-mail: yb@ysu.edu.cn.

Xiang-Dong Kong, born in 1959, is currently a professor at Yanshan University, China. He received his PhD degree from Yanshan University, China, in 1991. His main research interests include electro-hydraulic servo control system and robot control theory. Tel: +86-335-8051166; E-mail:xdkong@ysu.edu.cn.

Chun-He Li, born in 1989, is currently a master candidate at Yanshan University, China. His research interest is modeling in servo control system. E-mail: 345082951@qq.com.

Qi-Xin Zhu, born in 1992, is currently a master candidate at Yanshan University, China. His research interest is valve-controlled cylinder force control system. E-mail: 1755228049@qq.com.

Hua-Long Zhao, born in 1993, is currently a master candidate at Yanshan University, China. His research interest is robot control and modeling. E-mail: 731515432@qq.com.

Ling-Jian Kong, born in 1992, is currently a master candidate at Columbia University in the City of New York, USA. His research interest is robot architecture optimizes. E-mail: ljkonglicn@163.com. 


\section{Competing Interests}

The authors declare that they have no competing interests.

\section{Ethics Approval and Consent to Participate}

Not applicable.

\section{Funding}

Supported by National Natural Science Foundation of China (Grant No. 51605417), Key Project of Hebei Provincial Natural Science Foundation, China (Grant No. E2016203264), and State Key Laboratory of Fluid Power and Mechatronic Systems (Zhejiang University) Open Fund Project (Grant No. GZKF-201502)

\section{Publisher's Note}

Springer Nature remains neutral with regard to jurisdictional claims in published maps and institutional affiliations.

Received: 29 April 2016 Accepted: 15 April 2018

Published online: 11 May 2018

\section{References}

[1] Zhe Xu, Jun-Yao Gao, Hui Li, et al. The modeling and controlling of electrohydraulic actuator for quadruped robot based on fuzzy Proportion Integration Differentiation controller. Proceedings of the Institution of Mechanical Engineers, 2014, 228(14): 2557-2568.

[2] A Irawan, K Nonami. Friction effects on the load capacity of a column and a hydraulic cylinder. International Journal of Mechanical Sciences, 2009, 51(2): 145-151.

[3] Polkovnikov. Synthesis of the main parameters of servo actuators of hydraulic control surface drives of aircraft with a pump-controlled speed regulation. Teoriya i Sistemy Upravleniya, 2002, 41(4): 116-126.

[4] S Claudio. HyQ-design and development of a hydraulically actuated quadruped robot. University of Genoa, 2010.

[5] R Playter, M Buehler, M Raibert. BigDog. Proceedings of SPIE_The International Society for Optical Engineering, 2006, 6230II(2006).

[6] Zhou Ya, Yi-min Zhang, Xu-Fang Zhang, et al. Reliability sensitivity-based correlation coefficient calculation in structural reliability analysis. Chinese Journal of Mechanical Engineering, 2012, 25(3): 608-614.

[7] A Abolfaz, R Reza. Sensitivity analysis of influencing parameters in cavern stability. International Journal of Mining Science and Technology, 2012, 22(5): 707-710.

[8] Wei Liu, Gang Cao, Hong-Bo Zhai, et al. Sensitivity analysis and dynamic optimization design of supports' positions for engine pipelines. Journal of Aerospace Power, 2012, 237(2): 2756-2762.

[9] D Chatterjee, A Ghosh. Improvement of transient stability of power system with STATCOM-controller using trajectory sensitivity. International Journal of Electrical Power \& Energy Systems, 2011, 33(3): 531-539.

[10] Xiao-Meng Song, Jian-Yun Zhang, Chen-Sheng Zhan, et al. Global sensitivity analysis in hydrological modeling: Review of concepts, methods, theoretical framework, and applications. Journal of Hydrology, 2015, 523: 739-757.

[11] Wei Zheng, Hong-Hua Shi, Guo-Hong Fang, et al. Global sensitivity analysis of a marine ecosystem dynamic model of the Sanggou Bay. Ecological Modelling, 2012, 247(4): 83-94.

[12] A Saltelli, P Annoni, L Azzini, et al. Variance-based sensitivity analysis of model output. Design and estimator for the total sensitivity index. Computer Physics Communications, 2010, 181(2): 259-270.

[13] J W Hall, S A Boyce, Yue-Ling Wang, et al. Sensitivity analysis for hydraulic models. Journal of Hydraulic Engineering, 2009, 135(11): 959-969.

[14] R Bashar, W Ryder, G Angelis, et al. GPU-accelerated motion compensated OSEM list-mode PET reconstruction using a time-averaged sensitivity matrix. IEEE Nuclear Science Symposium \& Medical Imaging Conference, 2013: 1-4.

[15] K Billy, S Henry, T Stephen. Computation of the cycle state-variable sensitivity matrix of PWM DC/DC converters and its applications. IEEE Transactions on Circuits \& Systems I Fundamental Theory \& Applications, 2000, 47(10): 1542-1548.
[16] Li-Feng Zhang. Image reconstruction algorithm for electrical impedance tomography using updated sensitivity matrix. Soft Computing \& Pattern Recognition, 2011: 248-252.

[17] S Naseralavi, E Salajegheh, J Salajegheh, et al. Detection of damage in cyclic structures using an eigenpair sensitivity matrix. Computers \& Structures, 2012, 110-111(10): 43-59.

[18] M Vilenus. The application of sensitivity analysis to electrohydraulic position control servos. Universidad de Málaga, 2009, 105(2): 137-152.

[19] S Farasat, H Ajam. Sensitivity analysis of parameter changes in nonlinear hydraulic control systems. International Journal of Engineering, 2005, 18: 239-252.

[20] Xiang-Dong Kong, Bin Yu, Ling-Xiao Quan, et al. Trajectory sensitivity analysis of hydraulic drive unit of quadruped bionic robot. Journal of Mechanical Engineering, 2013, 49(14): 170-175.

[21] Xiang-Dong Kong, Bin Yu, Ling-Xiao Quan, et al. Nonlinear mathematical modeling and sensitivity analysis of hydraulic drive unit. Chinese Journal of Mechanical Engineering, 2015, 28(5): 999-111.

[22] Xiang-Dong Kong, Bin Yu, Ling-Xiao Quan, et al. Characteristic parameters sensitivity of position servo control for hydraulic drive unit of a quadruped robot in trotting gait. Robot, 2015, (1): 63-73.

[23] Xiang-Dong Kong, Kai-Xian Ba, Bin Yu, et al. Trajectory sensitivity analysis of first order and second order on position control system of highly integrated valve-controlled cylinder. Journal of Mechanical Science and Technology, 2015, 29(10): 4445-4464.

[24] Xiang-Dong Kong, Kai-Xian Ba, Bin Yu, et al. Force control compensation method with variable load stiffness and damping of the hydraulic drive unit force control system. Chinese Journal of Mechanical Engineering, 2016, 29(3): 454-464

[25] Qing-Liu Su, Fan-Dai Chao, Wang Lin, et al. Orthogonal test design for optimization of synthesis of MTX/LDHs hybrids by ion-exchange method. Journal of Physics \& Chemistry of Solids, 2015, 79: 82-88.

[26] Pei-Chao Zheng, Hong-Di Liu, Jin-Mei Wang, et al. Optimization of experimental conditions by orthogonal test design in a laser-induced breakdown experiment to analyze aluminum alloys. Analytical Methods, 2014, 6(7): 2163-2169.

\section{Submit your manuscript to a SpringerOpen ${ }^{\odot}$ journal and benefit from:}

- Convenient online submission

- Rigorous peer review

- Open access: articles freely available online

- High visibility within the field

- Retaining the copyright to your article

Submit your next manuscript at $\boldsymbol{\nabla}$ springeropen.com 